Check for updates

Cite this: Mater. Adv., 2022, 3,632

Received 10th September 2021 Accepted 3rd November 2021

DOI: 10.1039/d1ma00835h

rsc.li/materials-advances

\title{
An ionic hydrogel with stimuli-responsive, self-healable and injectable characteristics for the targeted and sustained delivery of doxorubicin in the treatment of breast cancer $t$
}

\author{
Muzammil Kuddushi, (D) $\ddagger^{\mathrm{a}}$ Deepak K. Pandey, (D) $\ddagger^{\mathrm{b}}$ Dheeraj K. Singh, (D) *b \\ Jitendra Mata ${ }^{c}$ and Naved Malek (DD *a
}

\begin{abstract}
Designing stimuli-responsive therapeutic scaffolds to deliver hydrophobic chemo drugs with controlled
and sustained release at the tumor site would be promising in cancer treatment. In a quest for the stimuli-responsive drug delivery system with sustained release, herein we have fabricated an active pharmaceutical ingredient-based ionic liquid (IL) based hydrogel with self-healable and injectable properties. The ionic hydrogel makes a localized, long-term co-delivery system with self-healable, injectable, and stimuli-responsive properties. Further, the smart hybrid ionic hydrogel was constructed through encapsulating doxorubicin (DOX) within the 3D matrix of the ionic hydrogel, which response to intracellular biological stimuli (e.g., $\mathrm{pH}$ and temperature) and releases DOX in $57 \mathrm{~h}$. Experimental results of IL-IL and IL-DOX interactions are supported through Density Functional Theory (DFT) calculations. In vitro cellular studies revealed that the DOX-loaded hybrid ionic hydrogel shows a synergistic anticancer effect against MCF-07 cells. These results demonstrate the ability of the ionic hydrogel as a promising candidate for local cancer therapeutics.
\end{abstract}

\section{Introduction}

Breast cancer, the most frequent cancer among women and impacting 2.1 million women every year, causes significant cancer-related deaths. ${ }^{1}$ The conventional treatment methods including surgery, radiotherapy, immunotherapy, and chemotherapy suffer side effects because of drug reactions, low therapeutic index, drug tolerance, and poor cancerous cell targeting. ${ }^{2}$ To circumvent these limitations, novel drug administration strategies including nano-drug carrier-based treatment such as liposomes, polymeric nanoparticles, surfactants and polymeric micelles, vesicles, dendrimers, hydrogels and recently discovered ionogels have been proposed. But they

\footnotetext{
${ }^{a}$ Ionic Liquids Research Laboratory, Department of Chemistry, Sardar Vallabhbhai National Institute of Technology, Surat-07, Gujarat, India.

E-mail: navedmalek@chem.svnit.ac.in; Tel: +9190 33167127

${ }^{b}$ Department of Basic Sciences, Institute of Infrastructure Technology Research and Management, Ahmedabad 380026, India

${ }^{c}$ Australian Centre for Neutron Scattering, Australian Nuclear Science and Technology Organization, Lucas Heights, NSW 2234, Australia

$\dagger$ Electronic supplementary information (ESI) available: Synthetic procedure of the IL with its characterization, SEM image of the ionic hydrogel, in vitro DOX release data from the ionic hydrogel and summary of the kinetics models applied for DOX release are reported. See DOI: 10.1039/d1ma00835h

\$ M. K. and D. K. P. share equal contribution
}

register slow drug release and irreversible deformation and therefore are not suitable for sustained and local administration. ${ }^{3}$ Hydrogels, despite significant progress, could be promising if they come with excellent biocompatibility, high mechanical properties, negligible cytotoxicity, high swelling properties, prominent drug-encapsulating capability and stimuli responsiveness. ${ }^{4-11}$ Ionic liquid (IL) based hydrogels, also known as ionic hydrogels, are the best alternatives because of their inherent properties that can be easily fine-tuned by judiciously selecting the constituting cations/anions of ILs. ${ }^{12-15}$

Polymeric hydrogels having 1-methyl-3-hexadecylimidazolium bromide as an additive with stimuli-responsive, self-healable, adhesive, and injectable properties are recently reported. When bromide was replaced by salicylate through metathesis with the sodium salicylate (NaSal), SAIL formed an ionic hydrogel at $4.45 \% \mathrm{w} / \mathrm{v}$ in an aqueous medium. ${ }^{9}$ We had selected a gelator concentration of $6.00 \%(\mathrm{w} / \mathrm{v})$ and solution $\mathrm{pH}$ of 7.4 for all the studies. NaSal is a non-steroidal antiinflammatory drug that can also activate mitogen-activated protein kinase that induces apoptosis in cancer cells. ${ }^{16,17}$ Zhao et al. reported salicylate-based hydrogels with integrated antifouling and antimicrobial capacities against Gram-negative Escherichia coli RP437 and Gram-positive Staphylococcus epidermidis. ${ }^{18}$ Kaule et al. used salicylate-based coatings in the drug-coated balloon catheter as it shows high paclitaxel 
encapsulation efficiency with a smooth surface and good aqueous solubility compared to the hyaluronic acid (bodyown hydrogel), and polyvinylpyrrolidone. ${ }^{19,20}$ Lin and coworkers reported thermo-responsive phase transition from a viscoelastic wormlike micelle solution to an elastic hydrogel in a mixture of 1-hexadecyl-3-methylimidazolium bromide, and NaSal. ${ }^{24}$ Zheng's group reported the fabrication of a $3 \mathrm{D}$ hydrogel from $N$-methyl- $N$-cetylpyrrolidinium bromide using NaSal as an external additive. ${ }^{21}$ Zhao et al. studied wormlike micelle formation by $N$-hexadecyl- $N$-methyl piperidinium bromide with the aid of NaSal. ${ }^{22}$ Raskova presented the effect exerted by the presence of NaSal in Carbomer-based hydrogel systems, which shows antimicrobial properties against Grampositive and Gram-negative bacterial strains, yeasts and moulds. $^{23}$

Combining different ionic functionalization to the judiciously composed structural architecture of the IL can specifically exploit the advantages of both, IL and solid polymer structures, enhancing the mechanical stability and improving the processability and durability of the hydrogels. ${ }^{12}$ Bearing in mind all above-described considerations and in the framework of our interest in obtaining supramolecular gels from ILs, herein we synthesized an active pharmaceutical ingredient based IL, i.e. 1-methyl-3-hexadecylimidazolium salicylate $\left(\mathrm{C}_{16} \mathrm{MeImSal}\right)$ as the LMWG. The $\mathrm{C}_{16} \mathrm{MeImSal}$ forms an ionic hydrogel that exhibited gel to sol transformation as a function of temperature. The ionic hydrogel was studied for the encapsulation and sustained release of the chemotherapeutic drug doxorubicin (DOX) and studied for its biological evaluation in breast cancer cell lines. ${ }^{25}$ The clinical application of DOX is restricted owing to its dose-dependent non-site-specific toxicity, such as cardio-toxicity and prevalence of off target effects is circumvented through hydrogel based new age drug delivery systems. Further, we used quantum chemical calculations through DFT to support our experimental results and to (i) obtain a greater insight into the intermolecular interactions in the hydrogel matrix, (ii) study the arrangements and interactions in the ionic hydrogel matrix and (iii) understand the DOXIL interactions. Structures of the $\mathrm{C}_{16} \mathrm{MeImSal}$ and DOX are drawn in Scheme 1.

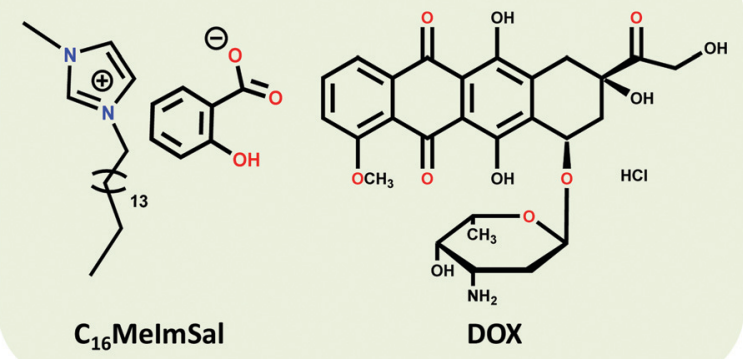

Scheme 1 Chemical structures of 1-methyl-3-hexadecylimidazolium salicylate $\left(\mathrm{C}_{16}\right.$ MelmSal) and doxorubicin hydrochloride (DOX).

\section{Materials and methods}

\section{Materials}

1-Methylimidazole ( $\geq 99.0 \%)$, 1-bromohexadecane $(97.0 \%)$ and doxorubicin hydrochloride (98.0-100.0\%) were procured from Sigma Aldrich. The MCF-07 cell line was obtained from NCCS, Pune, India. All solutions were prepared using Milli-Q water (Millipore) with an electrical resistivity of $18.2 \mathrm{M} \Omega \mathrm{cm}^{-1}$ at $25{ }^{\circ} \mathrm{C}$. For SAXS and NMR, samples were prepared in $\mathrm{D}_{2} \mathrm{O}$. $\mathrm{C}_{16} \mathrm{MeImSal}$ was synthesized and characterized as per a reported procedure that can be found in the ESI $\dagger$ (Scheme S1). ${ }^{9}$

\section{Fourier transform infrared spectroscopy (FTIR)}

The FTIR spectra of solid $\mathrm{C}_{16} \mathrm{MeImSal}$ and ionic hydrogel were recorded using a Shimadzu FTIR-8400S spectrophotometer.

\section{Morphology}

Morphology of the ionic hydrogel was studied through Scanning Electron Microscopy (SEM). For the detailed experimental description, see ref. 18.

\section{Small-angle X-ray scattering (SAXS)}

The SAXS measurements were performed on a Bruker NanoSTAR II SAXS instrument at ACNS, ANSTO. 1.541 A wavelength was used from a rotating anode $\mathrm{Cu} \mathrm{K} \alpha$ radiation source. Data were collected at a fixed detector distance, giving a $q$ range of 0.012-0.390 $\AA^{-1}$. Sealed quartz cells were used with a sample path length of $2 \mathrm{~mm}$ under vacuum. SAXS data were reduced at the instrument using Bruker software and then were analyzed using SasView software. SAXS data were analyzed using a cylinder model with the structure factor. The slope at low $q$ gives information about the possible structure that can be described through the form factor of a cylinder with a radius $R$ and a length $L$ as discussed for the transparent ionogel. ${ }^{26-28}$

The output of the scattering intensity function for randomly oriented cylinders is given by eqn (1) and (2): where the form factor $P(q)$

$$
P(q)=N \int_{0}^{\pi / 2} F^{2}(q) \sin (\gamma) \mathrm{d} \gamma
$$

with

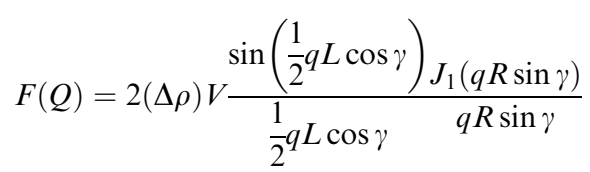

where $J_{1}$ is the first-order Bessel function and $\gamma$ is the angle between the axis of the cylinder and the $q$ vector, $V=\pi R^{2} L$ is the volume of the cylinder and $\Delta \rho$ (contrast) is the scattering length density difference between the scatterer and the solvent. The structure factor $S(q)$ was determined through SASView.

\section{Rheology}

A rheology test was carried out on a Physica MCR 301 (Anton Paar) rheometer at $25{ }^{\circ} \mathrm{C}$ with a $40 \mathrm{~mm}$-diameter parallel plate attached to a transducer. The gap was set to $1 \mathrm{~mm}$. The ionic 
hydrogel was placed between the parallel plate and the platform with special care to avoid evaporation of the solvent. Angular frequency sweep measurements were performed at 1-100 $\mathrm{rad} \mathrm{s}^{-1}$ under a constant strain of $1 \%$. These measurements were carried out within the viscoelastic region where $G^{\prime}$ and $G^{\prime \prime}$ are storage and loss modulus and are independent of strain amplitude. Strain scans were performed from 0.1 to $10 \%$ with a constant frequency of $1 \mathrm{rad} \mathrm{s}^{-1}$. The critical strain was quoted as the point that $G^{\prime}$ starts to deviate for linearity and ultimately crosses over $G^{\prime \prime}$, resulting in gel breakdown. Thixotropic properties or step strain sweep measurements were carried out to investigate the self-recovery properties of the hydrogels in response to applied shear forces. The strain was changed from $1 \%$ to $20 \%$ at the constant frequency value $(1.0 \mathrm{~Hz})$.

\section{Swelling behavior}

Ionic hydrogels with confirmed weights $\left(W_{\mathrm{a}}\right)$ were immersed in phosphate buffer solution (PBS) at pH 7.4 and pH $5.0\left(37^{\circ} \mathrm{C}\right)$.

\section{Dynamic light scattering (DLS) and zeta potential measurement}

DLS and zeta potential measurements were carried out in a Zetasizer Nano ZS (Malvern, UK) by varying the $\mathrm{pH}$ and temperature of the ionic hydrogel.

\section{Drug loading and encapsulation studies}

The ionic hydrogel was loaded with DOX using a modified breathing-in mechanism and put on a shaker for $3 \mathrm{~h}$. After loading, the solution was centrifuged at $10000 \mathrm{rpm}$ for 10 to 15 min. The supernatant obtained was diluted and the concentration of the drug in the supernatant was measured using a UV-Vis spectrometer at 480-485 $\mathrm{nm}$. Drug loading ratio and encapsulation efficiency of DOX in the ionic hydrogel were $15.12 \%$ and $93.20 \%$, respectively. Drug loading (DL) and encapsulation efficiencies (EE) were calculated using the following equation:

$$
\begin{gathered}
\operatorname{DL}(\%)=\frac{\text { Initial drug concentration }- \text { Drug concentration after loading }}{\text { Initial drug concentration }} \times 100 \\
\mathrm{EL}(\%)=\frac{\text { Loading efficiency }- \text { initial drug concentration }\left(\mu \mathrm{g} \mathrm{m} l^{-1}\right)}{\text { Initial hydrogel concentration }\left(\mu \mathrm{g} \mathrm{ml}^{-1}\right)} \times 100
\end{gathered}
$$

This procedure was repeated until no further weight increment occurred $\left(W_{\mathrm{b}}\right)$. At a pre-determined time, the ionic hydrogel was taken out and weighed. The weight of the swollen gel $\left(W_{\mathrm{X}}\right)$ was determined after removing the surface water gently with tissue paper. The swelling ratio (SR) of the hydrogel was calculated from the equation

$$
\mathrm{SR}=\frac{W_{\mathrm{a}}-W_{\mathrm{b}}}{W_{\mathrm{b}}}
$$

where $W_{\mathrm{a}}$ is the weight of water molecules in the swollen 3D ionic hydrogel network under certain conditions and $W_{\mathrm{b}}$ is the weight of the dry ionic hydrogel.

\section{Gel-to-sol transition}

Phase transition was confirmed through (i) turbidity measurements and (ii) tube inversion method. A phase transition was confirmed through two different procedures.

(i) The ionic hydrogel to ionic solution transition of hydrogels $(\mathrm{pH}=5.0)$ was studied upon increasing the temperature from 25 to $60{ }^{\circ} \mathrm{C}$ on a Cary $50 \mathrm{UV}$-Vis spectrometer with a Peltier-controlled variable temperature cell holder.

(ii) The tube-inverting experiment was used to measure the ionic hydrogel to ionic solution transition. Briefly, $1 \mathrm{~mL}$ ionic hydrogel was injected into $15 \mathrm{~mL}$ glass vials and equilibrated at $15{ }^{\circ} \mathrm{C}$ for $6 \mathrm{~h}$. Next, the glass vial containing the ionic hydrogel was immersed in a water bath at a given temperature for $5 \mathrm{~min}$ and then inverted $180^{\circ}$. If no visible flow was observed within $60 \mathrm{~s}$, the sample was considered as a gel. The observation was conducted from 25 to $60{ }^{\circ} \mathrm{C}$ with an increment of $1{ }^{\circ} \mathrm{C}$ each step.

\section{In vitro drug release study}

To measure the effect of temperature and $\mathrm{pH}$ changes on DOX release, the drug-loaded ionic hydrogel was immersed in $37{ }^{\circ} \mathrm{C}$; $\mathrm{pH}=5.0,37{ }^{\circ} \mathrm{C} ; \mathrm{pH}=7.4,25{ }^{\circ} \mathrm{C} ; \mathrm{pH}=5.0$, and $25{ }^{\circ} \mathrm{C} ; \mathrm{pH}=$ $7.4 \mathrm{PBS}$ at $150 \mathrm{rpm}$. At pre-determined time intervals, the drug release media were removed and substituted with fresh PBS. The amount of DOX concentration in the PBS release medium was measured with a UV-Vis spectrophotometer at 480-485 nm.

The release profiles of DOX were further analyzed based on mathematical drug release kinetics models. (i) Zero-order equation, (ii) first-order eqn and (iii) Higuchi release using the following plots: $M_{\mathrm{t}} v s . t, \log \left(M_{0}-M_{\mathrm{t}}\right)$ vs. $t$ and $M_{\mathrm{t}} v s$. square root of $t$, respectively.

\section{Confocal laser scanning microscopy detection}

The MCF-7 cells were seeded in confocal microscope (Leica Microsystems) dishes at $2 \times 10^{4}$ cells per well and cultured overnight. Then, the MCF-7 cells were exposed to bare DOX $\left(1 \mu \mathrm{g} \mathrm{mL} \mathrm{m}^{-1}\right)$ and DOX loaded ionic hydrogel for $4 \mathrm{~h}$, respectively. After the residual particles were removed, the cells were fixed with paraformaldehyde (4\%), and the cell nuclei were stained with $10 \mathrm{mM}$ 4,6-diamidino-2-phenylindole. Last, A Leica Microsystems confocal laser scanning microscope (Leica, Heidelberg, Germany) was utilized to capture the fluorescence images of the DOX $\left(1 \mu \mathrm{g} \mathrm{mL}{ }^{-1}\right)$ loaded ionic hydrogel.

\section{Cellular uptake}

To determine the cellular uptake of DOX and DOX loaded ionic hydrogel with MCF-7, cells were seeded in 6-well plates at $2 \times$ $10^{5}$ cells per well and cultured overnight. Then, the MCF-7 cells were treated with bare DOX and DOX $\left(1 \mu \mathrm{g} \mathrm{mL}^{-1}\right)$ loaded ionic 
hydrogel at the same DOX dose of $0.5 \mu \mathrm{g} \mathrm{mL}{ }^{-1}$. After $12 \mathrm{~h}$, cells were collected using centrifugation at $1000 \mathrm{rpm}$. Finally, the cells were suspended in $500 \mu \mathrm{L}$ PBS, and the cellular uptake of DOX was measured with an FACS Calibur flow cytometer (BD Biosciences, USA).

\section{In vitro cytotoxicity}

The cell viability by a neat ionic hydrogel, DOX, and DOX loaded ionic hydrogel was assessed using the 3-(4,5dimethylthiazol-2-yl)-2,5-diphenyltetrazolium bromide (MTT) assay on human breast cancer (MCF-7 cell lines). The cells were seeded at a density of $10^{4}$ cells per well in 96-well plates and cultured overnight. Subsequently, the cells were exposed to serial concentrations of DOX and DOX loaded hydrogel in culture medium, respectively. As controls, cells were incubated in a $5 \% \mathrm{CO}_{2}$ incubator at $37{ }^{\circ} \mathrm{C}$. After cultivation for $24 \mathrm{~h}$, the medium was removed, and the cells were washed carefully with PBS and incubated with fresh medium containing $5 \mathrm{mg} \mathrm{mL}$ MTT for another $4 \mathrm{~h}$. Medium was removed again, and DMSO (200 $\mu \mathrm{L}$ per well) was added. The plates were incubated at $37^{\circ} \mathrm{C}$ for $10 \mathrm{~min}$, and then the absorbance was recorded at $540 \mathrm{~nm}$. The $\mathrm{IC}_{50}$ values were calculated with GraphPad Prism Version 7.0 software (GraphPad Software, USA).

\section{Computational methodology}

The global minima on the potential energy surface of the $\mathrm{C}_{16} \mathrm{MeIm}^{+}$cation, $\mathrm{Sal}^{-}$anion, doxorubicin (DOX) molecule, $\mathrm{C}_{16} \mathrm{MeImSal}$ ionic liquid (IL), dimers of the IL and complex of the IL with DOX (IL-DOX) were computed by density functional theory (DFT) with the Gaussian 16 suite of software. ${ }^{29}$ A hybrid B3LYP functional with a $6-31 G^{* *}$ basis set in Gaussian 16 was utilized for the geometry relaxation. ${ }^{29,30}$ This $6-31 G^{* *}$ basis set with the B3LYP functional is an ideal level of theory to investigate the interaction mechanism in medium to large molecular systems and has previously also produced reliable and accurate results for a variety of molecular systems at minimum costs. $^{31-35}$ The frequency estimation at the same level of theory was performed with the aim to find out the true ground state geometries of studied systems (at $298.15 \mathrm{~K}$ and $1 \mathrm{~atm})^{36}$ and the optimized structures have been verified as a true minimum with no imaginary frequency. Dimers of the IL were used as a model of the ionic hydrogel. To investigate the nature and type of interactions in the hydrogel, the polarizable-continuum model with integral equation formalism (IEFPCM) was employed with water as a solvent as its dielectric constant $(\varepsilon)$ is 78.35. ${ }^{37}$ To investigate the contribution of weak interactions in these systems, Multiwfn 3.7 software was utilized to analyze the reduced density gradient (RDG) of IL and IL-DOX systems. ${ }^{38}$ The geometry of optimized structures and molecular electrostatic potential (MESP) maps were visualized using the GaussView 6 program $^{39}$ and Multiwfn ${ }^{38}$ with Visual Molecular Dynamics (VMD) ${ }^{40}$ 1.9.3 software was used for the RDG surface visualization.

Eqn (7) and (8) were used for the calculation of binding energies (BEs) of the IL and IL-DOX complex $(\Delta E)$ :

$$
\begin{gathered}
\Delta E_{\mathrm{IL}}=E_{\mathrm{IL}}-\left(E_{\text {cation }}+E_{\text {anion }}\right) \\
\Delta E_{\mathrm{IL}-\mathrm{DOX}}=E_{\mathrm{IL}-\mathrm{DOX}}-\left(E_{\mathrm{IL}}+E_{\mathrm{DOX}}\right)
\end{gathered}
$$

where $E_{\text {cation, }}, E_{\text {anion }}, E_{\mathrm{DOX}}, E_{\mathrm{IL}}$, and $E_{\mathrm{IL}-\mathrm{DOX}}$ are ground state optimized energies of cation, anion, DOX, IL and IL-DOX systems, respectively. The following equation was used to obtain the solvation energy of the IL:

$$
\Delta E_{\text {Solv }}=E_{\text {Sol }}-E_{\text {gas }}
$$

where $E_{\mathrm{Sol}}$ and $E_{\text {gas }}$ represent the total energies in the solution and gas phases, respectively. The values of chemical activity descriptors such as chemical hardness $(\eta),{ }^{41}$ chemical softness $(S),{ }^{41}$ chemical potential $(\mu),{ }^{41}$ and electrophilicity index $(\omega)^{42}$ were determined from the DFT calculation-based highest occupied molecular orbital (HOMO) and lowest unoccupied molecular orbital (LUMO) energies from the following equations:

$$
\begin{gathered}
\eta=\frac{I-A}{2} \\
S=\frac{1}{2 \eta} \\
\mu=-\frac{I+A}{2} \\
\omega=\frac{\mu^{2}}{2 \eta}
\end{gathered}
$$

where $I \approx-E_{\text {HOMO }}$ and $A \approx-E_{\text {LUMO }}$.

\section{Results and discussion}

\section{Molecular interactions within the ionic hydrogel}

Molecular interactions involved in the formation of the ionic hydrogel were studied through performing FTIR of the solid $\mathrm{C}_{16} \mathrm{MeImSal}$ and ionic hydrogel (Fig. S1, ESI $\dagger$ ). The frequency value of the $-\mathrm{C}=\mathrm{O}$ group at $1652 \mathrm{~cm}^{-1}$ for the powder sample is shifted to $1639 \mathrm{~cm}^{-1}$ for the ionic hydrogel because of hydration. The $\mathrm{C}-\mathrm{N}$ and $-\mathrm{C}=\mathrm{O}$ stretching vibrations, located at $1586 \mathrm{~cm}^{-1}$ and $1187 \mathrm{~cm}^{-1}$ in powder $\mathrm{C}_{16}$ MeImSal, are shifted to 1575 and $1174 \mathrm{~cm}^{-1}$ for the ionic hydrogel, respectively. These results suggest the electrostatic and hydrogen bonding interaction playing a dominant role in the formation of the ionic hydrogel. ${ }^{14,17,43}$

Further, we initiated quantum chemical calculations based on DFT to obtain a greater insight into the intermolecular interaction in the hydrogel matrix and contribution of weak non-covalent interactions in the drug-hydrogel complex. DFTbased quantum chemical calculations are employed in different fields of science to illustrate the electronic structures of various chemical species and have become an excellent method for addressing fascinating chemical challenges and mechanisms of reactions. Here, before investigating the interaction in the ionic hydrogel and drug-hydrogel complex, we first computed the ground state geometries of the isolated cation, anion and DOX. 


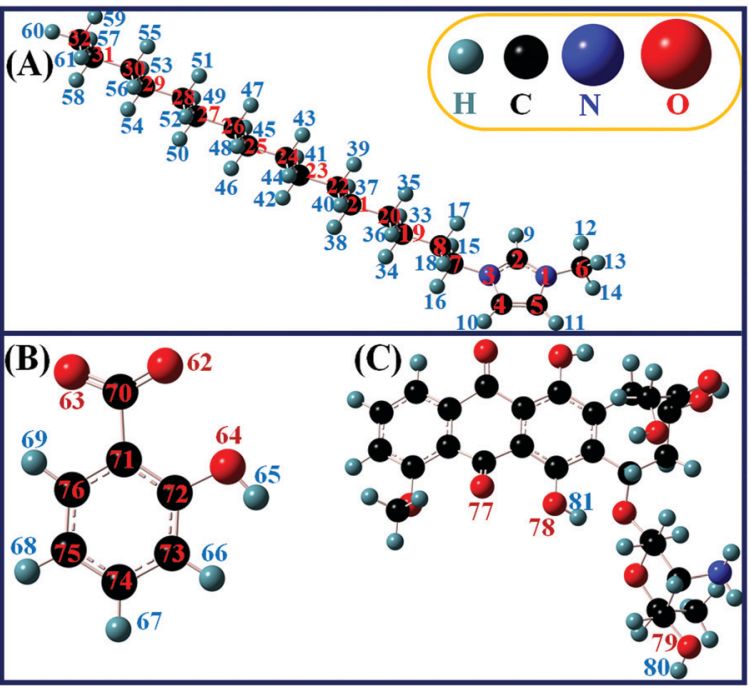

Fig. 1 The atomic numbering scheme for the DFT calculated and optimized structures of (A) the $\mathrm{C}_{16} \mathrm{Melm}^{+}$cation, (B) Sal anion and (C) DOX molecule.

The optimized structures are shown in Fig. 1 including the atomic numbering scheme. The $\mathrm{Sal}^{-}$anion is positioned at the $\mathrm{C}_{2}$ position of the $\mathrm{C}_{16} \mathrm{MeIm}^{+}$cation to obtain the minima on the potential energy landscape of $\mathrm{C}_{16} \mathrm{MeImSal}$, as it is well established that the $\mathrm{C}_{2}-\mathrm{H}_{9}$ position is the most acidic and important site for interaction with different anions and molecular systems in imidazolium cations. ${ }^{44-50,54,55}$ In order to investigate the strength of ion-pair interaction, the $\mathrm{BE}$ for $\mathrm{C}_{16}$ MeImSal was calculated. The calculated optimized energies of cations, anions, DOX, ILs (both in gas and solvent phases), the dimers of ILs and IL-Drug complex with BEs are listed in Table 1.

The BE for the IL $\left(-122.16 \mathrm{kcal} \mathrm{mol}^{-1}\right)$ demonstrates that $\mathrm{C}_{16} \mathrm{MeImSal}$ is energetically stable at the ground state. ${ }^{47,50}$ The optimized geometry of the IL demonstrates that the anion interacts with the cation through $\mathrm{C}-\mathrm{H} \cdots \mathrm{O}$ type hydrogen bonds (Fig. S2A, ESI $\dagger$ ). There are four hydrogen bonds between the cation and anion and one intramolecular hydrogen bond in the salicylate anion. Here, one can clearly distinguish the difference in the structure of the anion in both isolated form and ionpairs (Fig. 1B and Fig. S2A, ESI $\dagger$ ). A well-established criterion suggested by Arunan $e t$ al. for the determination of the strength

Table 1 DFT calculated optimized and binding energies of ground-state geometries using the B3LYP/6-31G** level of theory

\begin{tabular}{llll}
\hline Species & $\begin{array}{l}\text { Optimized energy } \\
\text { (Hartree) }\end{array}$ & BE (Hartree) & $\begin{array}{l}\mathrm{BE} \\
\left(\mathrm{kcal} \mathrm{mol}^{-1}\right)\end{array}$ \\
\hline $\mathrm{C}_{16} \mathrm{MeIm}^{+}$ & -894.999803 & - & - \\
$\mathrm{Sal}^{-}$ & -495.468729 & - & - \\
DOX & -1928.524047 & - & - \\
IL (gas phase) & -1390.663217 & -0.1946 & -122.16 \\
IL (solvent phase) & -1390.692045 & - & - \\
IL_DOX & -3319.266772 & -0.0795 & -49.89 \\
Dimer_1 & -2781.335071 & -0.0087 & -5.45 \\
Dimer_2 & -2781.362172 & -0.358 & -22.45 \\
Dimer_3 & -2781.345281 & -0.0189 & -11.85
\end{tabular}

of hydrogen bonding in ILs was adopted here. ${ }^{51}$ It is difficult, however, to classify the strength of a hydrogen bond; in the following, we consider an interaction to be strong if $r<2.3 \AA$ and weak for $2.3 \AA<r<2.6 \AA$. Such parameters have been chosen based on the $\mathrm{H} \cdots \mathrm{O}$ distances being shorter than the sum of the respective van der Waals radii. Based on this, the intramolecular hydrogen bond in the $\mathrm{Sal}^{-}$anion $\mathrm{O}_{64}-\mathrm{H}_{65} \cdots \mathrm{O}_{62}$ is found to be strong with a bond length of $1.59 \AA$. Similarly, the $\mathrm{C}_{2}-\mathrm{H}_{9} \cdots \mathrm{O}_{62}$ hydrogen bond between the cation and anion is found to be stronger in comparison to other hydrogen bonds between the cation and anion. Here, the Sal anion is also able to interact with both the methyl $\left(-\mathrm{CH}_{3}\right)$ and alkyl $-\mathrm{CH}_{2}$ group of the alkyl chain of the $\mathrm{C}_{16} \mathrm{MeIm}^{+}$cation by $\mathrm{C}-\mathrm{H} \cdots \mathrm{O}$ type of weak interaction, FTIR spectra vide supra (Fig. S2A, ESI $\dagger$ ). This case is considered to be comparable to that of experimental evidence available via the ${ }^{19} \mathrm{~F}$ NMR chemical shifts for alkyl chain interaction in $\mathrm{BF}_{4}$ based-ILs. ${ }^{52}$

To investigate various intra and intermolecular interactions involved in the ionic hydrogel formation of the $\mathrm{C}_{16} \mathrm{MeImSal}$ in aqueous media, we have studied the effect of solvation on the structure, energetics and reactivity of $\mathrm{C}_{16} \mathrm{MeImSal}$ in comparison to the gas phase. By simulating the surrounding of the $\mathrm{C}_{16} \mathrm{MeImSal}$ as a dielectric continuum with $\varepsilon=78.35$, using the IEFPCM model, the effect of an aqueous medium was analyzed. The solvation energy of the studied IL was $-18.09 \mathrm{kcal} \mathrm{mol}^{-1}$, indicating that solvation of the IL is a spontaneous process and suggesting that water increases the stability of the studied IL which is also clear from the more negative optimized energy of the IL in the solvent phase (Table 1). From Fig. S2B (ESI $\dagger$ ), it is clear that the $\mathrm{Sal}^{-}$anion slightly shifted towards the methyl group of the $\mathrm{C}_{16} \mathrm{MeIm}^{+}$cation in the solvent phase. In the Sal anion, the intramolecular hydrogen bond becomes stronger and the calculated bond length is $1.53 \AA$; similarly, the $\mathrm{C}_{6}-\mathrm{H}_{12} \cdots \mathrm{O}_{63}$ bond becomes stronger and the calculated bond length is found to be $1.90 \AA$. The vibrational spectra that can be used to assist the interpretation of experimental FTIR data have also been calculated here. The frequency values of the $-\mathrm{C}=\mathrm{O}$ group, which is at $1656 \mathrm{~cm}^{-1}$ for the gas phase, is shifted to $1646 \mathrm{~cm}^{-1}$ for the solvent phase i.e., ionic hydrogel, likely due to the hydration of the $-\mathrm{C}=\mathrm{O}$ group in the gel phase. The $\mathrm{C}-\mathrm{N}$ and $-\mathrm{C}-\mathrm{O}$ stretching vibrations, which are located at $1619 \mathrm{~cm}^{-1}$ and $1162 \mathrm{~cm}^{-1}$ in the gas phase of $\mathrm{C}_{16} \mathrm{MeImSal}$, are shifted to 1610 and $1159 \mathrm{~cm}^{-1}$ for the $\mathrm{C}_{16}$ MeImSal in the

Table 2 Observed FTIR vibrational bands of solid $\mathrm{C}_{16}$ MelmSal and ionic hydrogel with DFT computed vibrational bands of $\mathrm{C}_{16}$ MelmSal in gas and solvent phases

\begin{tabular}{lllll}
\hline & \multicolumn{2}{l}{$\begin{array}{l}\text { Observed wavenumber } \\
\left(\mathrm{cm}^{-1}\right)\end{array}$} & $\begin{array}{l}\text { Calculated wavenumber } \\
\left(\mathrm{cm}^{-1}\right)\end{array}$ \\
\cline { 2 - 3 } $\begin{array}{l}\text { Functional } \\
\text { group }\end{array}$ & $\mathrm{C}_{16}$ MeImSal & $\begin{array}{l}\text { Ionic } \\
\text { hydrogel }\end{array}$ & $\begin{array}{l}\mathrm{C}_{16} \text { MeImSal } \\
(\text { gas phase) }\end{array}$ & $\begin{array}{l}\mathrm{C}_{16} \text { MeImSal } \\
\text { (solvent phase) }\end{array}$ \\
\hline -C-N stretching & 1586 & 1575 & 1619 & 1610 \\
$-\mathrm{C}=\mathrm{O}$ & 1652 & 1639 & 1656 & 1646 \\
$-\mathrm{C}-\mathrm{O}-$ & 1187 & 1174 & 1162 & 1159 \\
$-\mathrm{OH}$ & - & 3348 & &
\end{tabular}


solvent phase, respectively (Table 2). Our DFT calculated IR spectra support the experimental findings of FTIR which predicts the dominant role of electrostatic interactions and hydrogen bonding in the formation of the three dimensional (3D) network structure of the ionic hydrogel. Here, it is very clear from the close inspection of Fig. S2A and B (ESI $\dagger$ ) that the DFT computations also predict the presence of inter- and intramolecular hydrogen bonding in $\mathrm{C}_{16} \mathrm{MeImSal}$, both in the gas and solvent phases.

It is interesting to explore the arrangements of the ionic hydrogel to understand the shape of the gelator. We studied the morphology of the ionic hydrogel through SEM. The SEM image (Fig. S3, ESI $\dagger$ ) shows the formation of a 3D branched fiberous network made of tightly winded cylindrical tubules. The ionic hydrogel is expected to be formed by encapsulating the water molecules within the network of the percolated gelator and inside the branched 3D structures. ${ }^{53}$

\section{Internal structure of the ionic hydrogel}

SAXS was further employed to verify the size and shape of the aggregates within the ionic hydrogel matrix. Fig. S4 (ESI $\dagger$ ) shows the experimental SAXS data of the ionic hydrogel. SAXS data clearly indicate the characteristic $q^{-1}$ behavior that is consistent with cylindrical shaped aggregates, SEM results vide supra. ${ }^{17}$ There is also a very strong structure factor peak at mid- $q .{ }^{15}$ A similar peak was reported in our earlier work with an imidazolium based ester functionalized ionic hydrogel. ${ }^{15}$ Local structures of the ionic hydrogel can be described by a cylinder of radius $2.10 \mathrm{~nm}$ and length $42.90 \mathrm{~nm}$. The cylindrical structure of the ionic hydrogel is due to the micelle like assembly (through hydrogen bonding and electrostatic interactions) between the long cation and anion of the $\mathrm{C}_{16} \mathrm{MeImSal}$ at the interiors of the micelles.

Structural optimization was performed for the $\mathrm{C}_{16} \mathrm{MeImSal}$ dimer using DFT computations to gain deeper insight into the arrangements and interactions in the ionic hydrogel. Without requiring extensive computing resources, a dimer may deliver valuable insights into the early-stage of aggregation. In parallel/ parallel-displaced or perpendicular or vertically tilted configurations, aromatic moieties can be aligned. ${ }^{56-58}$ The nitrogen atom containing imidazolium rings, however, is predicted to prefer a parallel or near-parallel configuration so that rings can enhance $\pi-\pi$ interactions along with the hydrogen bonding. The optimum arrangement of the dimer of $\mathrm{C}_{16} \mathrm{MeImSal}$ will be controlled by the interplay of interactions of mainly $\mathrm{H}$-bond and $\pi-\pi$ interactions between the imidazolium rings of the cation. Hence, in the present study, three configurations of dimers were prepared. In the first, cation rings are opposite sides of each other, in the second, both the rings are stacking in parallel and in the last, both the rings are in Y-shaped configuration.

Fig. 2 displays the ground state geometries of all the configurations of the dimers. Fig. $2 \mathrm{~B}$ presents the most stable dimer geometry with a $\mathrm{BE}$ of $-22.45 \mathrm{kcal} \mathrm{mol}^{-1}$ where two imidazolium rings are arranged in such a way that maximizes their $\pi-\pi$ interactions through parallel stacking and anion $-\pi^{+}$
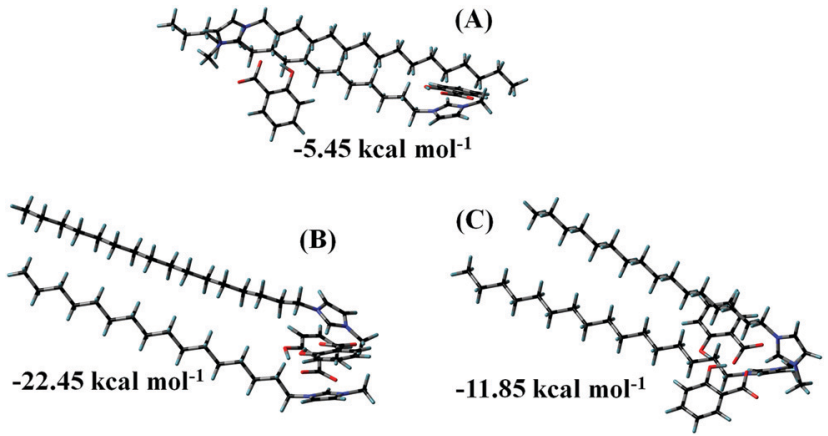

Fig. 2 Ground state geometries of dimer configurations along with their BEs. (A) Cation rings of $\mathrm{C}_{16} \mathrm{Melm}^{+}$are opposite, (B) cations rings are stacking in parallel, and (C) cation rings are in $\mathrm{Y}$-shaped configuration.

interactions in ion-pairs. The second most stable structure (Fig. 2C) in which the aromatic moieties are arranged perpendicular to each other (Y-shaped) is quite different from the structure in Fig. 2B and less stable. We predicted two lowest energy configurations of dimers, which can guide us to predict the arrangements of the ionic hydrogel to understand the formation and branching in the gel on the basis of dimer energetics. From Fig. $2 \mathrm{~B}$ and $\mathrm{C}$, one can notice that when the minimum energy structures are obtained, imidazolium rings are stacked and long alkyl chains are in parallel arrangements. Imidazolium rings take preferential on-top parallel orientations to increase the $\pi-\pi$ stacking that enhances the layering structure in ILs, despite the electrostatic repulsion and also hydrogen-bonding arrangements. This is the possible reason why the ionic hydrogel takes the cylindrical structure. ${ }^{59-61}$

\section{Mechanical properties of the ionic hydrogel}

The gel was subjected to the strain sweep and angular frequency sweep dynamic rheological test to explore its mechanical properties. At a fixed strain of $1 \%$, storage modulus $\left(G^{\prime}\right)$ was higher than loss modulus $\left(G^{\prime \prime}\right)$ within the whole frequency range (Fig. 3A), indicating the unstable colloidal particles that form a 3D network within the ionic hydrogel, SEM image, vide infra. The strain amplitude sweep of the hydrogel (Fig. 3B) demonstrated the elastic response typical of the polymeric hydrogel. Beyond the critical strain $\left(\gamma_{\mathrm{c}}\right)$ of $8.4 \%, G^{\prime}$ decreased rapidly, and the cross-over occurred at 9.3\%. Above $\gamma_{\mathrm{c}}, G^{\prime}$ decreases through breaking of the $3 \mathrm{D}$ network within the ionic hydrogel. The hydrogel studied here exhibits rapid recovery of its mechanical properties after a large amplitude oscillatory breakdown, known as thixotropic properties (Fig. 3C). ${ }^{62}$ Under the application of a large amplitude oscillatory force $(=20 \% ;=1$ $\operatorname{rad~s^{-1}}$ ), the $G^{\prime}$ decreases from 1949.23 to $525.61 \mathrm{~Pa}$, resulting in a quasi-liquid state of the hydrogel $(\tan \delta=0.46)$. However, when the amplitude is decreased $\left(=1 \% ;=1 \operatorname{rad~\mathrm {s}^{-1}}\right), G^{\prime}$ immediately recovers its initial value and returns to the quasi-solid state $(\tan \delta=0.49) .{ }^{63}$

According to the results of the rheological recovery property (Fig. 3C), the ionic hydrogel was converted to the solution state under higher shear strain and returned to the ionic hydrogel 


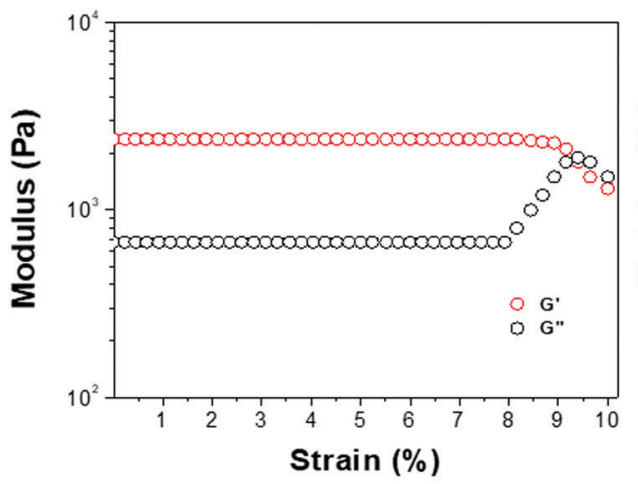

(A)

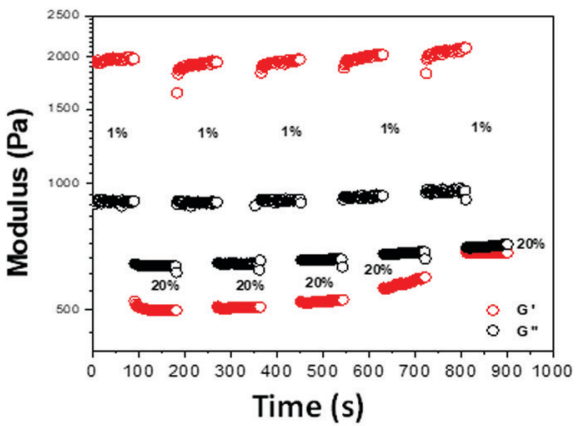

(C)

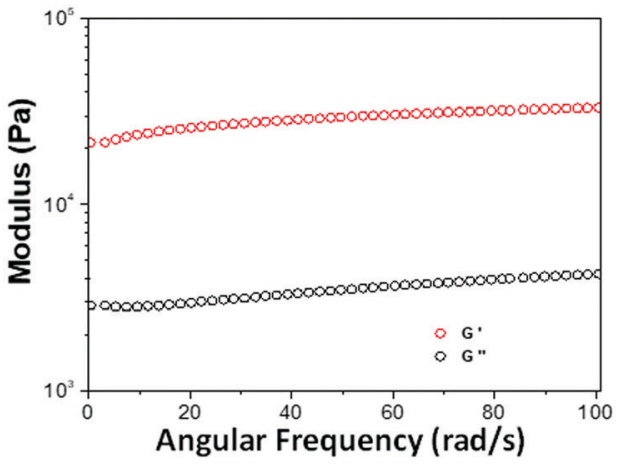

(B)
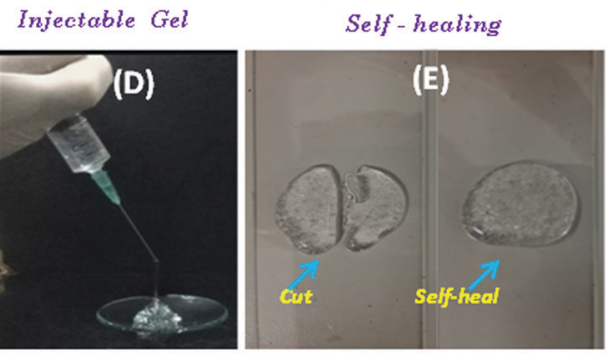

(D-E)

Fig. 3 (A) Strain sweep dynamic rheological data for the ionic hydrogel at $25^{\circ} \mathrm{C}\left(\omega=1 \mathrm{rad} \mathrm{s}^{-1}\right)$, (B) frequency sweep dynamic rheology data at $25^{\circ} \mathrm{C}$

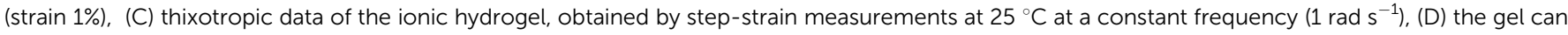
get through a 26-gauge needle smoothly without blockage, and (E) self-healing properties.

state when the stress was removed. Therefore, the ionic hydrogel could be used as an injectable gel for localized treatments. When the gel in the syringe was compressed, the pressure, which acted like a high dynamic strain, made the gel "flow" like a liquid to pass through the needle (Fig. 3D). This is due the dissociation of hydrogen bonds. Then the extruded gel returned to the gel state once the pressure was removed. The self-healing and injectable behaviours of the gel indicate that it may be used to deliver drugs and implanted in the body with a minimally invasive strategy. In addition, after the cut, within 5-7 minutes, the damaged gel could repair itself without any external stimulus (Fig. 3E), demonstrating the good self-healing abilities of the ionic hydrogel. ${ }^{64}$

\section{Swelling behavior of the ionic hydrogel}

The hydrogel (Fig. 4A) shows excellent water absorbing capacity; that is, the ionic hydrogel can absorb $42(\mathrm{pH}=7.4)$ and $70(\mathrm{pH}=5.0)$ times more water than its initial weight and take 280 and $320 \mathrm{~min}$ to reach a saturated state. The interactions of network components in ionic hydrogels restrain the whole matrix and limit the swelling ratio. The morphology of the ionic hydrogel directly influences its application as a drug

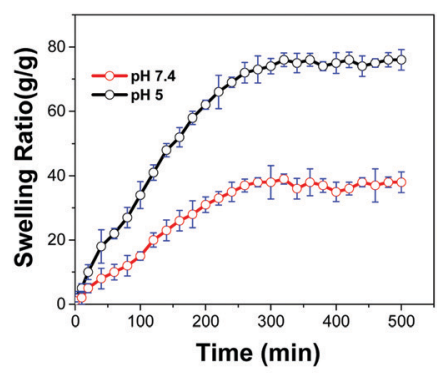

(A)
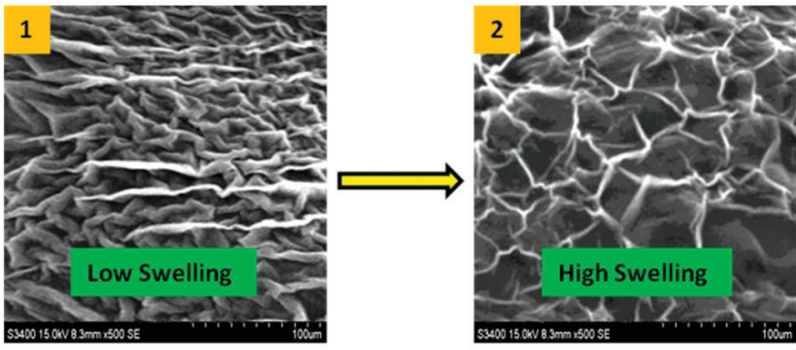

(B)

Fig. 4 (A) Swelling kinetics of the ionic hydrogel at $37^{\circ} \mathrm{C}$, and (B) SEM images of swollen ionic hydrogels at (i) $\mathrm{pH} 7.4$ and (ii) $\mathrm{pH} 5.0$ at $37{ }^{\circ} \mathrm{C}$. 
carrier, while the release mechanism will be affected by the mesh size. ${ }^{65,66}$

Both the ionic hydrogels showed porous $3 \mathrm{D}$ structures in PBS with different $\mathrm{pH}$ values at $37{ }^{\circ} \mathrm{C}$. The pore sizes of the ionic hydrogel in PBS with $\mathrm{pH} 5.0$ are higher than at $7.4 \mathrm{pH}$ (Fig. 4B). It might be due to the positive head group charge, which gets more protonated. The intermolecular electrostatic repulsion and enhanced hydrophilicity would make the ionic hydrogel swell considerably. ${ }^{67}$

\section{Size and zeta potential of the ionic hydrogel}

To effectively use the ionic hydrogel for targeted drug delivery, it is recommended to investigate whether the ionic hydrogel is stimuli responsive. To counter this argument, we hereby studied the hydrodynamic sizes of the gelators within the hydrogel matrix through DLS under the influence of temperature and $\mathrm{pH}$. The data also help us to define the conditions for the drug encapsulation and release. The ionic hydrogel was in more hydrated form and showed the maximum swelling state with the size of the aggregates in the range of $434 \pm 5 \mathrm{~nm}$ at $25{ }^{\circ} \mathrm{C}$ (Table 3 and Fig. 5). However, as the temperature rises to $37^{\circ} \mathrm{C}$, the size of the aggregates within the hydrogel decreases to $403 \pm 5 \mathrm{~nm}$, which may be due to the exclusion of water molecules from the hydration shell. This eventually resulted in the collapse of the hydrogel due to volume phase transition (VPT) and resulted in a more compact gel network because of the increased hydrophobicity. ${ }^{65,68}$ It is worth mentioning here that PDI values decrease with temperature $\left(0.24\right.$ at $25{ }^{\circ} \mathrm{C}$ and 0.12 at $37^{\circ} \mathrm{C}$ ), indicative of the phase transition (Table 3).

Table 3 Temperature and $\mathrm{pH}$ dependent size distribution of ionic hydrogels

\begin{tabular}{llllllll}
\hline $\begin{array}{l}\text { Ionic } \\
\text { hydrogel }\end{array}$ & 25 & \multicolumn{2}{l}{ Temperature $\left({ }^{\circ} \mathrm{C}\right)$} & & $\mathrm{pH}\left(25^{\circ} \mathrm{C}\right)$ & & $\mathrm{pH}\left(37^{\circ} \mathrm{C}\right)$ \\
\hline Size & $437 \pm 5$ & $403 \pm 5$ & $530 \pm 5$ & $496 \pm 5$ & $640 \pm 5$ & $580 \pm 5$ \\
PDI & 0.24 & 0.12 & 0.21 & 0.28 & & 0.29 & 0.20
\end{tabular}

Further, zeta potential data of the hydrogel with and without DOX exhibited a minor change in the surface charge from +22.5 to $+21.5 \mathrm{mV}$ (without DOX), -17.4 to $-19.7 \mathrm{mV}$ (with DOX-25 ${ }^{\circ} \mathrm{C}, \mathrm{pH}=5$ ), and -11.2 to $-14.3 \mathrm{mV}$ (with $\mathrm{DOX}-37{ }^{\circ} \mathrm{C}, \mathrm{pH}=7.4$ ) across the temperature range that indicates the cationic ionic hydrogel stability with temperature (Fig. 5B). ${ }^{69}$

An inverse correlation was observed between the temperature and $\mathrm{pH}$ on the size of aggregates within the hydrogel. The interplay of forces responsible for swelling of the hydrogel is certainly responsible for the changes observed at higher temperature and acidic pH (Fig. 5 and Table 3). At pH 5.0, the ionic hydrogel gets protonated and attracts a higher amount of water molecules on its surface through H-bonding and increases the size of the aggregates. Higher degree of swelling at acidic $\mathrm{pH}$ further strengthens the claim, vide infra (Fig. 5). It was found that the ionic hydrogel has the desired characteristics with an optimum size in response to temperature $\left(37^{\circ} \mathrm{C}\right)$ and $\mathrm{pH}(5.0)$ as shown in Table 3, while the rest of the other formulations did not show any particular trend. These data are the basis of our drug encapsulation and release of the entrapped drug from the ionic hydrogel.

\section{DOX-IL interaction studies through DFT calculations}

After investigating the role of weak non-covalent interactions in the formation of the ionic hydrogel (IL in both gas and solvent phases and dimer), we studied the drug-hydrogel interaction through DFT calculations. It has been observed that when molecules vary considerably in their sizes, the geometric mixing concept seems insufficient, as is the scenario in the present research. ${ }^{70,71}$ Using DFT computations, we computed the binary interplay between the IL and DOX to resolve this constraint. Ideally, to measure this sort of interactions, one will conduct a thorough molecular dynamics (MD) simulation, but this would be an excessively time-consuming process. In addition, classical simulations are based on the empirical force fields and their accuracy and reliability can often be suspected unless the accuracy of the force field is confirmed by substantial experimental results. $^{72}$ For this justification, we have adopted a

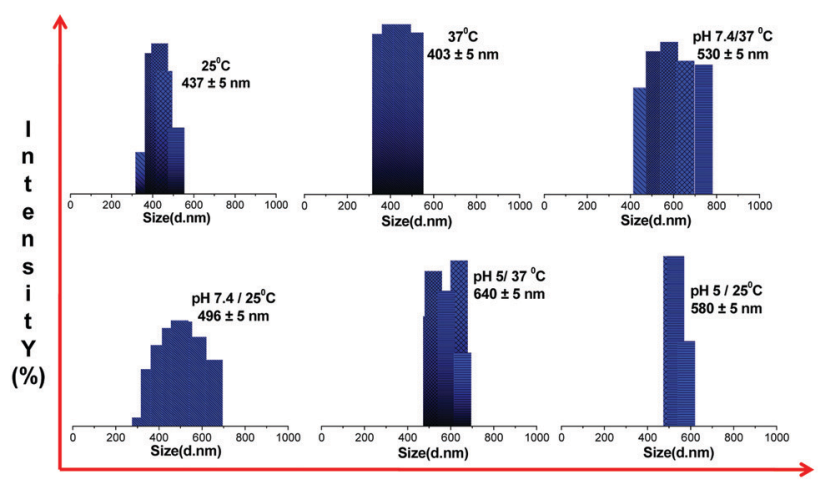

(A)

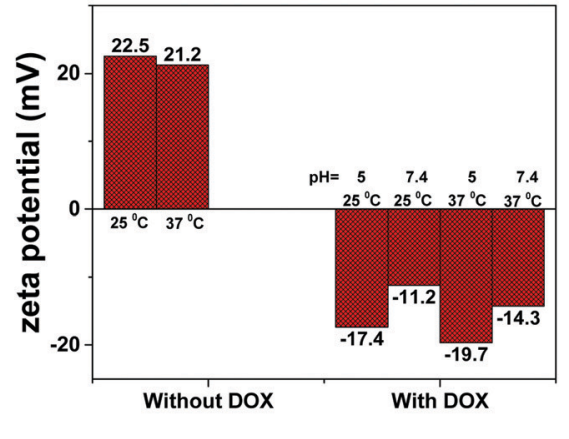

(B)

Fig. 5 Temperature and $\mathrm{pH}$ dependent size distribution of ionic hydrogels. 
methodology that does not depend on empirical force fields and does not necessitate substantial computing facilities. Hence, in order to investigate such interactions in the drughydrogel complex, the interaction between the single unit of the IL and the drug (DOX) molecule was carried out to mimic the experimental results. The optimized structure of the ILDOX complex is presented in Fig. S2C (ESI $\dagger$ ). The ground-state structure of the complex reveals that the molecule of the DOX interacts with the IL by hydrogen bonds of type $\mathrm{C}-\mathrm{H} \cdots \mathrm{O}$, where the DOX acts as a hydrogen-bonding donor. The drug molecule interacts with the IL by two $\mathrm{C}-\mathrm{H} \cdots \mathrm{O}$ (with cation) and one $\mathrm{O}-\mathrm{H} \cdots \mathrm{O}$ (with anion) type hydrogen bonds i.e. $\mathrm{C}_{6}-\mathrm{H}_{13} \cdots \mathrm{O}_{78}$, $\mathrm{C}_{5}-\mathrm{H}_{11} \cdots \mathrm{O}_{77}$ and $\mathrm{O}_{79}-\mathrm{H}_{80} \cdots \mathrm{O}_{63}$ with bond distances of $2.29 \AA$, $2.76 \AA$ and $1.69 \AA$, respectively (Fig. S2C, ESI $\dagger$ ). We have calculated the $\mathrm{BE}$ for this complex in the gas phase and the value is $-49.89 \mathrm{kcal} \mathrm{mol}^{-1}$ (Table 1 ). The negative BE value suggests that the mechanism of complex formation is thermodynamically stable and demonstrates the stability of the IL-DOX complex. Our computed BE value for the IL-DOX complex suggests that the DOX drug will bind to the carrier of the hydrogel (IL), but this interaction is not so strong as to prevent the drug at the target site from being desorbed. For adsorption and drug delivery, the binding energy of $\sim 30 \mathrm{kcal} \mathrm{mol}^{-1}$ is appropriate. ${ }^{73}$ Our calculated DFT results, however, only demonstrate the drug-hydrogel complex interaction mechanism and do not illustrate the temperature and $\mathrm{pH}$-dependent characteristics, but are able to provide deeper insights into the mechanism of interaction. The primary purpose of our research is to better understand the mechanism of interaction, so we will address the formation of weak non-covalent interactions in these systems by examining the molecular electrostatic potentials (MESP) and reduced density gradient (RDG).

\section{Electronic properties}

To gain insight into the relationship between molecule structure and activity, MESP were computed. Due to electron density distribution, the MESP is represented as the potential encountered by a unit positive charge close to the molecular system. The MESP directs the quantitative polarity of the molecule; electrophilic attack sites can be predicted by a positive potential, while nucleophilic attack sites are indicated by a negative potential. The expression for ESP is given by the following equation at any point $r$ in the space near the molecule: ${ }^{74,75}$

$$
V(r)=\sum \frac{Z_{\mathrm{A}}}{\left|R_{\mathrm{A}}-r\right|}-\int \frac{\rho_{\left(r^{\prime}\right) \mathrm{d} r^{\prime}}}{\left|r^{\prime}-r\right|}
$$

where the charge on the nucleus $A$ observed at $R_{\mathrm{A}}$ is $Z_{\mathrm{A}}$ and the electron density is $\rho(r)$.

$V(r)$ describes the net electrostatic effect of the molecule's total charge distribution (electrons + nuclei) at point $r$. Negative ESPS depict the nucleophilic sites of attack (red colour) on the molecular surface in Fig. S5 (ESI $\dagger$ ), whereas positive ESPs show the electrophilic sites (blue colour). Green colour is used for zero-potential surfaces. The entire surface of the imidazolium ring is shown in Fig. S5A (ESI $\dagger$ ) to be blue and light blue can be observed over the alkyl chain, reflecting the most positive ESPS. While red colour is present near $-\mathrm{C}=\mathrm{O}$ and $-\mathrm{OH}$ groups in the isolated $\mathrm{Sal}^{-}$anion, yellow colour is present in the entire ring area, indicating the presence of negative ESPS (Fig. S5B, ESI $\dagger$ ). Charge re-distribution occurs after ion-pair formation, resulting in a mixture of red, blue, yellow, and green colours. The red colour is only present on the oxygen atoms and blue colour on the cation in Fig. S5D (ESI $\dagger$ ), which shows the possibility of the strong interaction between these two moieties by the bifurcated $\mathrm{C}-\mathrm{H} \cdots \mathrm{O}$ type non-covalent bonds. The red colour on the $-\mathrm{OH}$ group also justifies the experimental finding that predicts the intramolecular hydrogen bonding in the Sal anion. In addition, all the colours are present in the DOX molecule and the red colour can be seen near the $-\mathrm{C}=\mathrm{O}$ and $-\mathrm{OH}$ group, while the blue colour can be seen on the other - $\mathrm{OH}$ group linked to the ring-containing $-\mathrm{NH}_{2}$ group. In the drug-hydrogel complex, the presence of blue colour on these -OH groups increases the possibility of strong hydrogen bonding. The red colour of the drug molecule (DOX) on $\mathrm{O}_{79}$ provides it with an opportunity to interact with the hydrogel, which further confirms the findings of the structural component determined by DFT and thus confirms the experimental findings.

Further, we have evaluated the values of HOMO and LUMO energy levels to calculate the chemical reactivity descriptors such as chemical potential $(\mu)$, softness $(S)$, hardness $(\eta)$, and electrophilicity-index $(\omega)$. The significant chemical-reactivity descriptors $(\mu, S, \eta$, and $\omega)$ including the dipole moment for the hydrogel (IL) carrier, drug molecule (DOX), and IL-DOX complex are provided in Table 4. In terms of these descriptors, the electronic properties and stability such as electron-transfer in a complex molecule are demonstrated quite well. Significant aspects of the IL-DOX complex have been observed. The complex IL-DOX was softer $\left(0.47 \mathrm{eV}^{-1}\right)$ than the IL $\left(0.25 \mathrm{eV}^{-1}\right)$ and DOX molecules $\left(0.28 \mathrm{eV}^{-1}\right)$, which is illustrated by the higher chemical softness and lower values of chemical hardness (Table 4). Compared to the chemical hardness $\eta$ of IL $(1.99 \mathrm{eV})$ and DOX molecules $(1.72 \mathrm{eV})$, the chemical hardness of the IL-DOX complex $(1.04 \mathrm{eV})$ was lower. The chemical potential of the complex IL-DOX $(-4.01 \mathrm{eV})$ was greater than that of the molecules of IL $(-2.73 \mathrm{eV})$ and DOX $(-4.00 \mathrm{eV})$, with a higher negative chemical potential of the complex implying the stability of the complex. The dipole moment of the complex increased from the $4.44 \mathrm{D}$ of the drug molecule (DOX) to 8.24 D, which corresponds to increasing solubility as well. The electrophilicity-index assesses the energy decrease associated

Table $4 \mathrm{HOMO}$ and LUMO energy levels, band gap energies $E_{\mathrm{g}}$, dipole moment $p$, chemical potential $(\mu)$, chemical softness $(S)$, chemical hardness $(\eta)$, and electrophilicity index $(\omega)$ of $C_{16}$ MelmSal, DOX and $C_{16}$ MelmSal-DOX complex in the gas phase

\begin{tabular}{lllllllll}
\hline & $\begin{array}{l}E_{\text {HOMO }} \\
(\mathrm{eV})\end{array}$ & $\begin{array}{l}E_{\mathrm{LUMO}} \\
(\mathrm{eV})\end{array}$ & $\begin{array}{l}E_{\mathrm{g}} \\
(\mathrm{eV})\end{array}$ & $\begin{array}{l}\text { Dipole } \\
\text { moment } p\end{array}$ & $\begin{array}{l}\eta \\
(\mathrm{eV})\end{array}$ & $\begin{array}{l}S \\
\left(\mathrm{eV}^{-1}\right)\end{array}$ & $\begin{array}{l}\mu \\
(\mathrm{eV})\end{array}$ & $\begin{array}{l}\omega \\
(\mathrm{eV})\end{array}$ \\
\hline IL & -4.73 & -0.73 & 3.99 & 12.81 & 1.99 & 0.25 & -2.73 & 1.86 \\
DOX & -5.73 & -2.28 & 3.44 & 4.44 & 1.72 & 0.28 & -4.00 & 4.65 \\
IL-DOX & -5.05 & -2.96 & 2.08 & 8.24 & 1.04 & 0.47 & -4.01 & 7.71
\end{tabular}


with a complex system obtaining additional charges from the surroundings. The chemical reactivity parameters described above, such as lower hardness, greater electrophilicity-index value, high charge-transfer and negative chemical potential of the ILDOX complex, clearly demonstrate that this complex can be used as an efficient cancer therapy drug delivery system.

\section{Non-covalent interaction (NCI) analysis}

To envisage the non-covalent interactions (NCI) such as hydrogen bonding, van der Waals interactions, and steric-repulsions among the non-covalently interacting systems, NCI analysis is an essential tool. This approach is based on electronic densities and their derivatives, such as reduced density gradients (RDGs). ${ }^{76}$ The RDG function can be defined using the following formula:

$$
\operatorname{RDG}(r)=\frac{1}{2\left(3 \pi^{2}\right)^{\frac{1}{3}}} \frac{|\Delta \rho(r)|}{\rho(r)^{\frac{4}{3}}}
$$

where $\rho(r)$ is the electron density.

Weak non-covalent interactions play an important role in the determination of the physicochemical properties in ILs and their derived hydrogel systems. The NCI visual representation of the IL is shown in Fig. S6 (ESI $\dagger$ ) and is plotted between the $\operatorname{sign}\left(\lambda_{2}\right) \rho$ and reduced-density gradient (RDG). It depicts the presence of strong hydrogen bonding (H-bond) and weak van der Waals interactions between the $\mathrm{C}_{16} \mathrm{MeIm}{ }^{+}$cation and anion. An essential parameter employed to detect the existence of chemical bonding in the complex is the $\operatorname{sign}\left(\lambda_{2}\right) \rho$ in the scatter plot of NCI analysis. The values of $\operatorname{sign}\left(\lambda_{2}\right) \rho$ below zero indicate hydrogen bonding and beyond zero display steric repulsions and spikes around the zero regions of $\operatorname{sign}\left(\lambda_{2}\right) \rho$ indicate van der Waals interactions. The 3D-colour filled isosurface for the IL is depicted in Fig. 6A, which also illustrates the existence of non-covalent interactions between cation and anion moieties. The presence of blue colour between $\mathrm{C}_{2}-\mathrm{H}_{9}$ and $\mathrm{O}_{62}$; and $\mathrm{O}_{64}-\mathrm{H}_{65}$ and $\mathrm{O}_{62}$ atoms shows the area where intermolecular and intramolecular hydrogen bonding takes place, and the areas of weak van der Waals forces and strong steric repulsion in the imidazolium and $\mathrm{Sal}^{-}$anion rings are indicated by green and red colour, respectively.

Similarly, Fig. 6B presents the NCI visual representation, which depicts the presence of weak non-covalent interactions in the drug-hydrogel complex (IL-DOX complex). Surprisingly, for the IL-DOX complex, all categories of NCIs were observed, viz weak van der Waals forces, hydrogen bonding, and stericrepulsions. The NCI between the IL and DOX molecule are also demonstrated by the 3D colour-filled iso-surface presented in Fig. 6B. Van-der Waals forces are shown by green-colour between the IL and DOX, while the blue-region occurs due to hydrogen bonding interactions. These interactions are highly in line with the $\mathrm{C}-\mathrm{H} \cdots \mathrm{O}$ and $\mathrm{O}-\mathrm{H} \cdots \mathrm{O}$ type weak interactions observed in the geometrical analysis of IL and IL-DOX complexes.

In this way, our DFT calculation-based NCI analysis is perfectly able to demonstrate the contribution of interactions

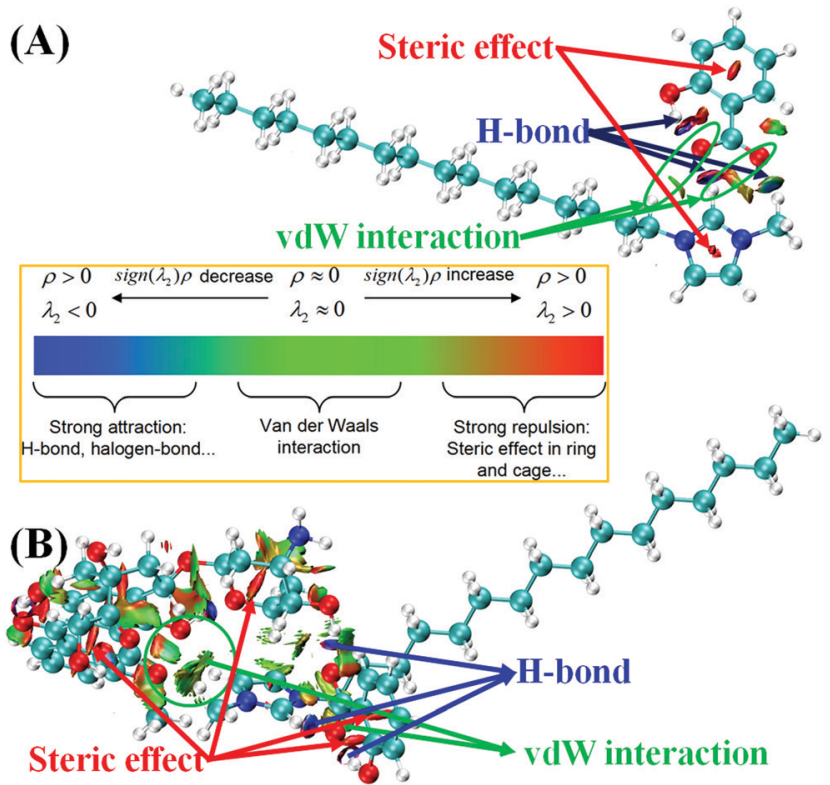

Fig. 6 3D colour filled RDG iso-surfaces of (A) $C_{16}$ MelmSal, and (B) $\mathrm{C}_{16}$ MelmSal-DOX complex showing the nature of the interaction between the cation-anion, and $\mathrm{C}_{16} \mathrm{MelmSal}$ and DOX molecule.

in the hydrogel and IL-DOX complex. In addition, the presence of weak drug-hydrogel carrier interactions plays a key role in drug-off loading at the target site. Further, these low-cost computational calculations by considering only single ionpair (IL), DOX and IL-DOX molecule allowed us to see what an immediate site-specific interaction would be like.

\section{Gel-to-sol transition}

The ionic hydrogel exhibited semi solid like behavior and nonflowing characteristics with almost zero transmittance at 25 to $40{ }^{\circ} \mathrm{C}(\mathrm{pH}=5.0)$ (Fig. S7, ESI $\left.\dagger\right)$. With increasing the temperature from 40 to $60{ }^{\circ} \mathrm{C}$, the transmittance increased gradually, and at $47{ }^{\circ} \mathrm{C}$ the ionic hydrogel transformed into a transparent solution. From the tube inversion method, we observed ionic hydrogel-to-solution transition at a temperature of $47 \pm 2{ }^{\circ} \mathrm{C}$ (Fig. S7B, ESI $\dagger$ ). Such a phase transition was reversible and is probably because of the association and disassociation of the non-covalent interaction between IL-IL when the temperature and $\mathrm{pH}$ were changed. ${ }^{77}$

\section{In vitro drug release study}

At $37{ }^{\circ} \mathrm{C}$ and $5.0 \mathrm{pH}$, the \% of release of DOX from the ionic hydrogel was well-controlled and lasted for over $57 \mathrm{~h}$ (Fig. S8, ESI $\dagger$ ). No fast DOX release was observed at the initial stage, and the $\%$ of release was over $\sim 86.4 \%$ at the later stage. The $\%$ of release was found to be around $\sim 45.3 \%$ at $\mathrm{pH} 7.4$ over a period of $57 \mathrm{~h}$. Similar results were observed when the hydrogel was incubated at $25{ }^{\circ} \mathrm{C}$. The cumulative DOX release was $\sim 33.2 \%$ at $\mathrm{pH} 5.0$ and $\sim 18.2 \%$ at $\mathrm{pH}$ 7.4. As expected, the acidic $\mathrm{pH}$ increased the release process of DOX, attributed to the increased hydrophilicity of DOX and de-solubilization inside the ionic hydrogel fibrous network. Further, more drug was 


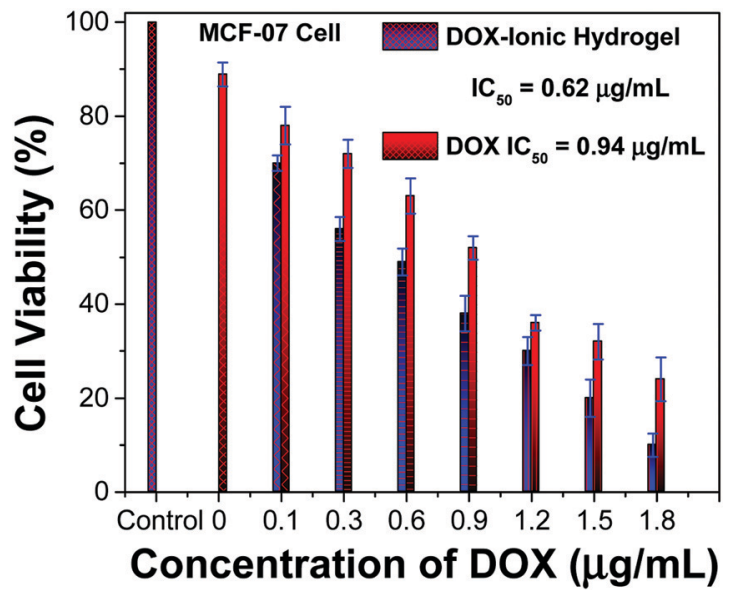

Fig. 7 In vitro cytotoxicity study of the neat ionic hydrogel, DOX, and DOX loaded ionic hydrogel.

released at $37{ }^{\circ} \mathrm{C}$, likely due to the increased permeability within the hydrogel network. ${ }^{78}$ In conclusion, these smart ionic hydrogels demonstrate a release of anticancer drugs controlled by dual stimuli, temperature and $\mathrm{pH}^{42}$

Taking them altogether, the \% of DOX release from the ionic hydrogel system $\left(37^{\circ} \mathrm{C}, \mathrm{pH}\right.$ 5.0) exhibited a sustained codelivery approach as long as $57 \mathrm{~h}$, and the \% release amounts of the DOX exceeded $86.4 \%$. The release data were further evaluated via mathematical models of the drug release kinetics
(Table S1, ESI $\dagger$ ). The results showed that the release of DOX from the ionic hydrogel system was well-fitted by the Higuchi release equation with good correlation coefficients $\left(R^{2} \approx 0.97\right) .{ }^{79}$ The presence of stimuli $\left(5.0 \mathrm{pH}\right.$ and $\left.37{ }^{\circ} \mathrm{C}\right)$ in the ionic hydrogel network resulted in intermolecular interactions and affected the DOX release mechanisms.

\section{In vitro cytotoxicity study}

The cell viability study suggested that the neat ionic hydrogel had excellent biocompatibility and no cytotoxicity. The data are in good agreement with the MCF-07 cell line assay, while neat DOX and DOX loaded ionic hydrogels indicate that they could induce tumor cell death and inhibit cell growth. Further, the $\mathrm{IC}_{50}$ values were determined from the dose response curve for neat DOX and DOX loaded ionic hydrogels against MCF-7 cell lines using GraphPad Prism Software (Fig. 7). Regardless of incubation time, the percentage of living cells in the presence of the DOX loaded ionic hydrogel was lower than that of neat DOX. This could be due to the slow release of DOX from the ionic hydrogel network. The $\mathrm{IC}_{50}$ values in the cases of neat DOX and DOX loaded polymeric hydrogels for MCF-07 cells were found to be 0.94 and $0.62 \mu \mathrm{g} \mathrm{mL} \mathrm{L}^{-1}$ at $24 \mathrm{~h}$ of the exposure. Apparently, the loaded ionic hydrogels exhibited good anti-cancer activity with a lower IC $_{50}$ value than the neat DOX in MCF-7 cells.

\section{DOX uptake study}

The cellular uptake of neat DOX and DOX loaded ionic hydrogel in the MCF-07 cell line, depicted in MCF-07 cells, was viewed

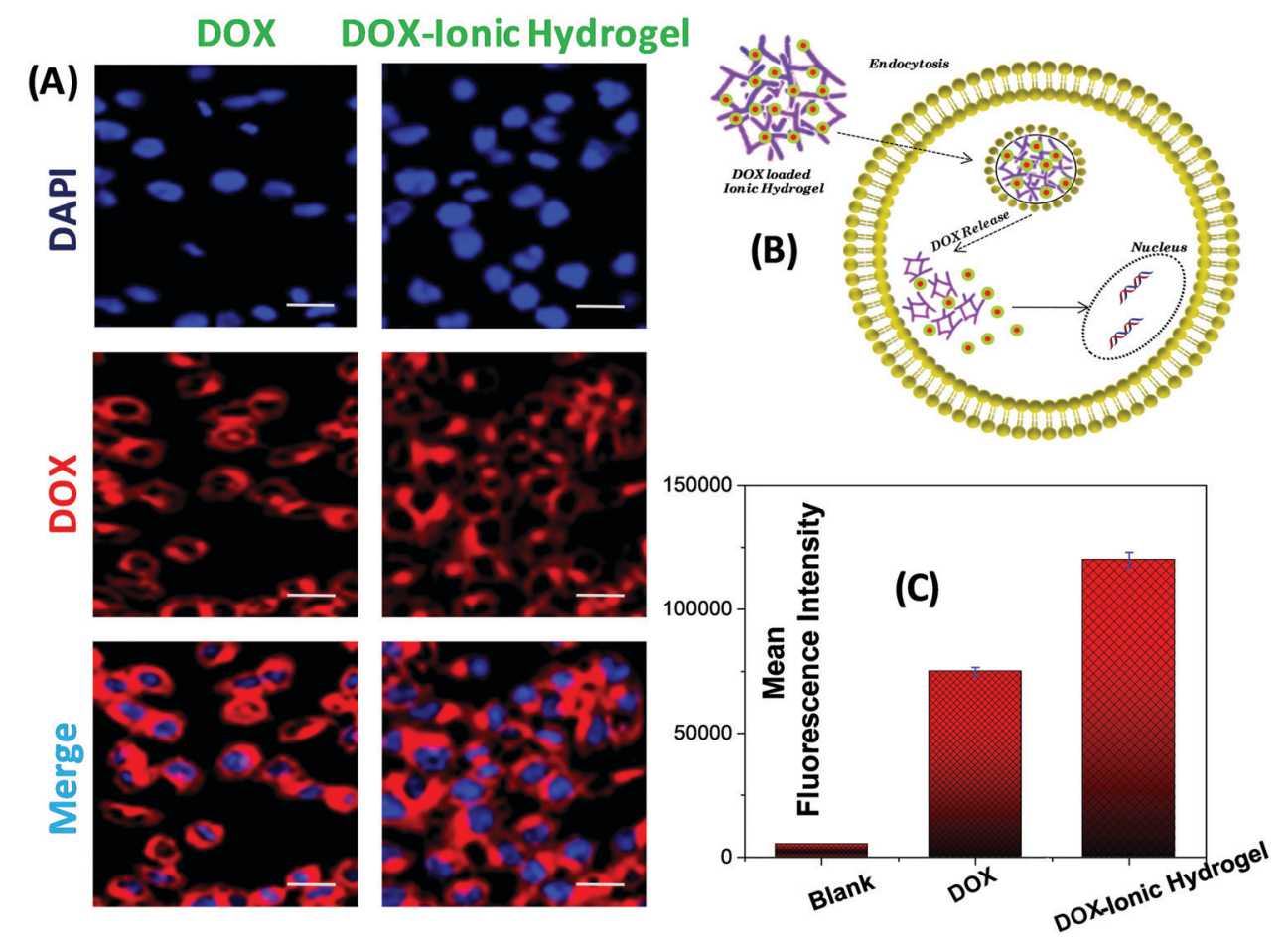

Fig. 8 (A) Representative fluorescence images of MCF-07 cells incubated with DOX, and DOX loaded ionic hydrogel at an equivalent DOX concentration of $1 \mu \mathrm{g} \mathrm{mL}^{-1}$ for $4 \mathrm{~h}$; scale bar is $20 \mu \mathrm{m}$, (B) quantitative measurement of DOX uptake in MCF-07 cells by flow cytometric analysis, and (C) the mechanism for enhancing DOX delivery. 
with a confocal laser scanning microscope (CLSM) where the cell nuclei exhibited strong blue fluorescence (Fig. 8A). The red fluorescence of DOX was clearly seen in the cytoplasm and nuclei of MCF-07 cells, which are prepared with neat DOX and DOX-ionic hydrogel. The uptake of DOX in the DOX loaded ionic hydrogel was higher than that of the neat DOX. This may be a result of the difference in the cellular uptake mechanism. ${ }^{80}$ The cellular uptake of neat DOX is through passive diffusion, while the ionic hydrogel transports into cells by both endocytosis and passive diffusion. The combination of the two pathways might explain the high uptake of the DOX-ionic hydrogel. In summary, the DOX-ionic hydrogel could help the DOX enter the cells and to be released from the 3D network of the ionic hydrogel efficiently (Fig. 8B), which was consistent with the above results obtained by laser scanning confocal microscopy. To quantify the cell uptake, we performed flow cytometry measurements. Fig. 8C clearly shows a remarkably increased cellular uptake and greatly enhanced nucleus localization in cells treated with the DOX loaded ionic hydrogel with more systematic intracellular DOX release. Evidently, the DOX loaded ionic hydrogel has appreciable possibilities to achieve cellspecific drug delivery.

\section{Conclusions}

Herein, we designed a local drug administrative system for the poorly water-soluble drug DOX using an amphiphilic ionic hydrogel as the stimuli-responsive and injectable drug carrier. The hydrogel was successfully realized as a sustained codelivery system for the DOX. DOX could be effectively encapsulated into the cores of cylindrical micelles formed by the amphiphilic hydrogel in water and released from the ionic hydrogel formulation in a well-controlled and sustained manner at $37{ }^{\circ} \mathrm{C}$ and $\mathrm{pH}$ 5. In vitro cytotoxicity and uptake, results confirmed a synergistic anticancer effect of the DOX-delivery system against the human breast cancer cell line (MCF-7). Here, we have employed the DFT calculations, which helped us to investigate the interaction mechanism in the ionic hydrogel and drug-hydrogel interaction. Overall, this stimuli responsive ionic hydrogel provides an ideal drug career for breast cancer treatment.

\section{Conflicts of interest}

There are no conflicts to declare.

\section{Acknowledgements}

M. K. and D. K. S. acknowledge UGC-DAE-CSR and SERB-DST ECR for the financial assistance under UDCSR/MUM/AO/CRSM-276/2017 and ECR/2016/001289. D. K. P. acknowledges DST, India, for providing financial support under the INSPIRE Fellowship No. IF170625. The authors appreciate the support of the ACNS, ANSTO, Australia, in providing the SAXS beamtime (Proposal No. 6210). Research Grant for Research Promotion under Technical Education-STEM is kindly acknowledged.

\section{References}

1 https://www.who.int/cancer/prevention/diagnosis-screening/ breast-cancer/en/.

2 S. Senapati, A. K. Mahanta, S. Kumar and P. Maiti, Controlled drug delivery vehicles for cancer treatment and their performance, Signal Transduction and Targeted Ther., 2018, 3(1), 7.

3 M. Sepantafar, R. Maheronnaghsh, H. Mohammadi, F. Radmanesh, M. M. Hasani-Sadrabadi, M. Ebrahimi and H. Baharvand, Engineered hydrogels in cancer therapy and diagnosis, Trends Biotechnol., 2017, 35(11), 1074-1087.

$4 \mathrm{~J}$. Li and D. J. Mooney, Designing hydrogels for controlled drug delivery, Nat. Rev. Mater., 2016, 1, 16071.

5 Q. Jin, Z. Xin, X. M. Peter and G. Baolin, pH-responsive selfhealing injectable hydrogel based on N-carboxyethyl chitosan for hepatocellular carcinoma therapy, Acta. Biomater., 2017, 58, 168-180.

6 L. Yongping, Z. Xin, X. M. Peter, G. Baolin, D. Yaping and H. Xuezhe, pH-responsive injectable hydrogels with mucosal adhesiveness based on chitosan-grafted-dihydrocaffeic acid and oxidized pullulan for localized drug delivery, J. Colloid Interface Sci., 2019, 536, 224-234.

7 Y. Gao, J. Peng, M. Zhou, Y. Yang, X. Wang, J. Wang, Y. Cao, W. Wang and D. Wu, A multi-model, large range and antifreezing sensor based on a multi-crosslinked poly(vinyl alcohol) hydrogel for human-motion monitoring, J. Mater. Chem. B, 2020, 8, 11010-11020.

8 M. Norouzi, B. Nazari and D. W. Miller, Injectable hydrogelbased drug delivery systems for local cancer therapy, Drug Discovery Today, 2016, 21(11), 1835-1849.

9 M. Kuddushi, N. K. Patel, J. P. Mata, O. El Seoud and N. I. Malek, Imidazole-based surface-active gelator: Thermo responsive gel-to-gel transition of 1-hexadecyl-3-methyl imidazolium salicylate for multidimensional applications, J. Mol. Liq., 2021, 117773.

10 B. Balakrishnan, N. Joshi, K. Thorat, S. Kaur, R. Chandan and R. Banerjee, A Tumor Responsive Self Healing Prodrug Hydrogel Enables Synergistic Action of Doxorubicin and Miltefosine for Focal Combination Chemotherapy, J. Mater. Chem. B, 2019, 7(18), 2920-2925.

11 J. Claus, A. Brietzke, C. Lehnert, S. Oschatz, N. Grabow and U. Kragl, Swelling Characteristics and Biocompatibility of Ionic Liquid Based Hydrogels for Biomedical Applications, PLoS One, 2020, 15(4), 1-16.

12 L. Chaunier, L. Viau, X. Falourd, D. Lourdin and E. Leroy, A drug delivery system obtained by hot-melt processing of zein plasticized by a pharmaceutically active ionic liquid, J. Mater. Chem. B, 2020, 8, 4672-4679.

13 M. Kuddushi, S. Rajput, A. Shah, J. Mata, V. K. Aswal, O. El Seoud, A. Kumar and N. I. Malek, Stimuli Responsive, SelfSustainable, and Self-Healable Functionalized Hydrogel with Dual Gelation, Load-Bearing, and Dye-Absorbing Properties, ACS Appl. Mater. Interfaces, 2019, 11(21), 19572-19583.

14 M. Kuddushi, J. Mata and N. Malek, Self-Sustainable, SelfHealable, Load Bearable and Moldable Stimuli Responsive 
Ionogel for the Selective Removal of Anionic Dyes from Aqueous Medium, J. Mol. Liq., 2019, 298, 112048.

15 M. Kuddushi, N. K. Patel, S. L. Gawali, J. P. Mata, H. MontesCampos, L. M. Varela, P. A. Hassan and N. I. Malek, Thermo-Switchable de Novo Ionogel as Metal Absorbing and Curcumin Loaded Smart Bandage Material, J. Mol. Liq., 2020, 306, 112922.

16 A. G. P. Pinto, G. P. D. Ribeiro, M. O. Azevedo, J. E. Cunha, K. Bica, M. Vasiloiu, S. Reisa, S. Reis and L. M. F. Saraiva, Ionic Liquids: insights into the Evaluation of Pharmaceutical Profiles, New J. Chem., 2013, 4095-4102.

17 M. Kuddushi, N. K. Patel, S. Rajput, O. A. El Seoud, J. Mata and N. I. Malek, Temperature Responsive Low Molecular Weight Ionic Liquid Based Gelator: An Approach to Fabricate Anti-Cancer Drug Loaded Hybrid Ionogel, ChemSystemsChem, 2020, 2, e1900053.

18 C. Zhao, X. Li, L. Li, G. Cheng, X. Gong and J. Zheng, Dual Functionality of Antimicrobial and Antifouling of Poly $(n-$ Hydroxyethylacrylamide)/Salicylate Hydrogels, Langmuir, 2013, 29(5), 1517-1524.

19 S. Kaule, I. Minrath, F. Stein, U. Kragl and W. Schmidt, Correlating Coating Characteristics with the Performance of Drug-Coated Balloons - A Comparative In Vitro Investigation of Own Established Hydrogel- and Ionic Liquid-Based Coating Matrices, PLoS One, 2015, 1-18.

20 S. Petersen, S. Kaule, F. Stein, I. Minrath and K. Schmitz, Novel Paclitaxel-Coated Angioplasty Balloon Catheter Based on Cetylpyridinium Salicylate: Preparation, Characterization and Simulated Use in an in Vitro Vessel Model, Mater. Sci. Eng., C, 2013, 33(7), 4244-4250.

21 H. Yan, M. Zhao and L. Zheng, Colloids Hydrogel Formed by Cetylpyrrolidinium Bromide and Sodium Salicylate, Colloids Surf., A, 2011, 392(1), 205-212.

22 M. Zhao, Z. Yan, C. Dai, M. Du, H. Li, Y. Zhao, K. Wang and Q. Ding, Formation and Rheological Properties of Wormlike Micelles by $N$-Hexadecyl- $N$-Methylpiperidinium Bromide and Sodium Salicylate, Colloid Polym. Sci., 2015, 293(4), 1073-1082.

23 Z. Kolarova Raskova, M. Hrabalikova and V. Sedlarik, Effect of Sodium Salicylate on the Viscoelastic Properties and Stability of Polyacrylate-Based Hydrogels for Medical Applications, Int. J. Polym. Sci., 2016, 2016, 1-6.

24 Y. Lin, Y. Qiao, Y. Yan and J. Huang, Thermo-Responsive Viscoelastic Wormlike Micelle to Elastic Hydrogel Transition in Dual-Component Systems, Soft Matter, 2009, 5(16), 3047-3053.

25 J. Gehl, M. Boesgaard, T. Paaske, B. Vittrup Jensen and P. Dombernowsky, Combined doxorubicin and paclitaxel in advanced breast cancer: Effective and cardiotoxic, Ann. Oncology, 1996, 7(7), 687-693.

26 A. Shah, M. Kuddushi, D. Ray, V. K. Aswal and N. I. Malek, Sodium Salicylate Mediated Ionic Liquid Based Catanionic Coacervates as Membrane-Free Microreactors for the Selective Sequestration of Dyes and Curcumin, ChemSystemsChem, 2020, 2(3), e1900029.

27 M. Kuddushi, J. Mata and N. Malek, Self-Sustainable, SelfHealable, Load Bearable and Moldable Stimuli Responsive
Ionogel for the Selective Removal of Anionic Dyes from Aqueous Medium, J. Mol. Liq., 2019, 298, 112048.

28 K. Wood, J. P. Mata, C. J. Garvey, C. M. Wu, W. A. Hamilton, P. Abbeywick, D. Bartlett, F. Bartsch, P. Baxter and N. Booth, et al., QUOKKA, the Pinhole Small-Angle Neutron Scattering Instrument at the OPAL Research Reactor, Australia: Design, Performance, Operation and Scientific Highlights, J. Appl. Crystallogr., 2018, 51(2), 294-314.

29 M. Frisch, G. Trucks, H. Schlegel, G. Scuseria, M. Robb, J. Cheeseman, G. Scalmani, V. Barone, G. Petersson and H. Nakatsuji, et al., Gaussian 16 Rev. B. 01, Wallingford, CT. 2016.

30 A. D. Becke, Density-Functional Exchange-Energy Approximation with Correct Asymptotic Behavior, Phys. Rev. A: At., Mol., Opt. Phys., 1988, 38, 3098.

31 R. Khorram, H. Raissi and A. Morsali, Assessment of Solvent Effects on the Interaction of Carmustine Drug with the Pristine and Cooh-Functionalized Single-Walled Carbon Nanotubes: A Dft Perspective, J. Mol. Liq., 2017, 240, 87-97.

32 H. Shaki, H. Raissi, F. Mollania and H. Hashemzadeh, Modeling the Interaction between Anti-Cancer Drug Penicillamine and Pristine and Functionalized Carbon Nanotubes for Medical Applications: Density Functional Theory Investigation and a Molecular Dynamics Simulation, J. Biomol. Struct. Dyn., 2019, 1-13.

33 M. Shahabi and H. Raissi, Comprehensive Theoretical Prediction of the Dynamics and Stability Properties of Tegafur Pharmaceutical Agent on the Graphene Based Nanostructures in Aqueous Environment, Appl. Surf. Sci., 2018, 455, 32-36.

34 M. Zaboli and H. Raissi, A Combined Molecular Dynamics Simulation and Quantum Mechanics Study on Mercaptopurine Interaction with the Cucurbit [6,7] Urils: Analysis of Electronic Structure, Spectrochim. Acta, Part A, 2018, 188, 647-658.

35 A. Alinejad, H. Raissi and H. Hashemzadeh, Understanding Co-Loading of Doxorubicin and Camptothecin on Graphene and Folic Acid-Conjugated Graphene for Targeting Drug Delivery: Classical Md Simulation and Dft Calculation, J. Biomol. Struct. Dyn., 2020, 38, 2737-2745.

36 D. A. McQuarrie, Statistical Mechanics, Amer Inst Physics, 1305 Walt Whitman RD, Ste 300, Melville, NY, USA, pp. 11747-4501, 1965.

37 E. Cances, B. Mennucci and J. Tomasi, A New Integral Equation Formalism for the Polarizable Continuum Model: Theoretical Background and Applications to Isotropic and Anisotropic Dielectrics, J. Chem. Phys., 1997, 107, 3032-3041.

38 T. Lu and F. Chen, Multiwfn: A Multifunctional Wavefunction Analyzer, J. Comput. Chem., 2012, 33, 580-592.

39 R. Dennington, T. A. Keith, J. M. Millam, K. Eppinnett, L. W. Hovell and R. Gilliland, GaussView, Version 6, Semichem Inc., Shawnee Mission, KS, 2016.

40 W. Humphrey, A. Dalke and K. Schulten, Vmd: Visual Molecular Dynamics, J. Mol. Graph, 1996, 14, 33-38.

41 R. G. Parr and R. G. Pearson, Absolute Hardness: Companion Parameter to Absolute Electronegativity, J. Am. Chem. Soc., 1983, 105, 7512-7516. 
42 R. G. Parr, L. V. Szentpaly and S. Liu, Electrophilicity Index, J. Am. Chem. Soc., 1999, 121, 1922-1924.

43 S. M. Hashemnejad and S. Kundu, Probing Gelation and Rheological Behavior of a Self-Assembled Molecular Gel, Langmuir, 2017, 33, 7769-7779.

44 K. Dong, Y. Song, X. Liu, W. Cheng, X. Yao and S. Zhang, Understanding Structures and Hydrogen Bonds of Ionic Liquids at the Electronic Level, J. Phys. Chem. B, 2012, 116, 1007-1017.

45 K. Noack, P. S. Schulz, N. Paape, J. Kiefer, P. Wasserscheid and A. Leipertz, The Role of the $\mathrm{C} 2$ Position in Interionic Interactions of Imidazolium Based Ionic Liquids: A Vibrational and Nmr Spectroscopic Study, Phys. Chem. Chem. Phys., 2010, 12, 14153-14161.

46 D. K. Singh, B. Rathke, J. Kiefer and A. Materny, Molecular Structure and Interactions in the Ionic Liquid 1-Ethyl-3Methylimidazolium Trifluoromethanesulfonate, J. Phys. Chem. A, 2016, 120, 6274-6286.

47 P. Sanchora, D. K. Pandey, D. Rana, A. Materny and D. K. Singh, Impact of Size and Electronegativity of Halide Anions on Hydrogen Bonds and Properties of 1-Ethyl-3Methylimidazolium-Based Ionic Liquids, J. Phys. Chem. A, 2019, 123, 4948-4963.

48 D. K. Singh, P. Donfack, B. Rathke, J. Kiefer and A. Materny, Interplay of Different Moieties in the Studied by Raman Spectroscopy and Density Functional Theory Calculations, J. Phys. Chem. B, 2019, 123, 4004-4016.

49 D. K. Singh, S. Cha, D. Nam, H. Cheong, S. W. Joo and D. Kim, Raman Spectroscopic Study on Alkyl Chain Conformation in 1-Butyl-3-Methylimidazolium Ionic Liquids and Their Aqueous Mixtures, ChemPhysChem, 2016, 17, 3040-3046.

50 D. K. Pandey, P. Sanchora, D. Rana, P. Donfack, A. Materny and D. K. Singh, Impact of Water on the Hydrogen Bonding between Halide-Based Ion-Pairs Investigated by Raman Scattering and Density Functional Theory Calculations, J. Raman Spectrosc., 2020, 51, 147-164.

51 E. Arunan, G. R. Desiraju, R. A. Klein, J. Sadlej, S. Scheiner, I. Alkorta, D. C. Clary, R. H. Crabtree, J. J. Dannenberg and P. Hobza, Definition of the Hydrogen Bond (Iupac Recommendations 2011), Pure Appl. Chem., 2011, 83, 1637-1641.

52 A. Khrizman, H. Y. Cheng, G. Bottini and G. Moyna, Observation of Aliphatic $\mathrm{C}-\mathrm{H} \cdots \mathrm{X}$ Hydrogen Bonds in Imidazolium Ionic Liquids, Chem. Commun., 2015, 51, 3193-3195.

53 S. M. Rajput, U. U. More, Z. S. Vaid, K. D. Prajapati and N. I. Malek, Impact of Organic Solvents on the Micellization and Interfacial Behavior of Ionic Liquid Based Surfactants, Colloids Surf., A, 2016, 507, 182-189.

54 D. K. Pandey, H. L. Kagdada, A. Materny and D. K. Singh, Hybrid Structure of Ionic Liquid and $\mathrm{TiO}_{2}$ Nanoclusters for Efficient Hydrogen Evolution Reaction, J. Phys. Chem. A, 2021, 125(12), 2653-2665.

55 D. K. Pandey, H. L. Kagdada, A. Materny and D. K. Singh, Hybrid Structure of Ionic Liquid and ZnO Nano Clusters for Potential Application in Dye-Sensitized Solar Cells, J. Mol. Liq., 2020, 114538.
56 M. Pitonak, P. Neogrady, J. Rezac, P. Jurecka, M. Urban and P. Hobza, Benzene Dimer: High-Level Wave Function and Density Functional Theory Calculations, J. Chem. Theory Comput., 2008, 4, 1829-1834.

57 Y. C. Park and J. S. Lee, Accurate Ab Initio Binding Energies of the Benzene Dimer, J. Phys. Chem. A, 2006, 110, 5091-5095.

58 T. F. Headen, C. A. Howard, N. T. Skipper, M. A. Wilkinson, D. T. Bowron and A. K. Soper, Structure of $\pi-\pi$ Interactions in Aromatic Liquids, J. Am. Chem. Soc., 2010, 132, 5735-5742.

59 F. Tang, T. Ohto, T. Hasegawa, M. Bonn and Y. Nagata, $\pi-\pi$ Stacking of Imidazolium Cations Enhances Molecular Layering of Room Temperature Ionic Liquids At Their Interfaces, Phys. Chem. Chem. Phys., 2017, 19(4), 2850-2856.

60 R. P. Matthews, T. Welton and P. A. Hunt, Competitive Pi Interactions and Hydrogen Bonding within Imidazolium Ionic Liquids, Phys. Chem. Chem. Phys., 2014, 16(7), 3238-3253.

61 Y. L. Wang, A. Laaksonen and M. D. Fayer, Hydrogen Bonding versus $\pi-\pi$ Stacking Interactions in ImidazoliumOxalatoborate Ionic Liquid, J. Phys. Chem. B, 2017, 121(29), 7173-7179.

62 Z. Li, Y. M. Zhang, H. Y. Wang, H. Li and Y. Liu, Mechanical Behaviors of Highly Swollen Supramolecular Hydrogels Mediated by Pseudorotaxanes, Macromolecules, 2017, 50(3), 1141-1146.

63 W. Xie, Q. Gao, Z. Guo, D. Wang, F. Gao, X. Wang, Y. Wei and L. Zhao, An Injectable and Self-Healing ThermoSensitive Magnetic Hydrogel for Asynchronous Control Release of Doxorubicin and Docetaxel to Treat TripleNegative Breast Cancer, ACS Appl. Mater. Interfaces, 2017, 9(39), 33660-33673.

64 M. Li, W. Li, W. Cai, X. Zhang, Z. Wang, J. Street, W. J. Ong, Z. Xia and Q. Xu, A Self-Healing Hydrogel with Pressure Sensitive Photoluminescence for Remote Force Measurement and Healing Assessment, Mater. Horiz., 2019, 6(4), $703-710$.

65 N. K. Verma, M. P. Purohit, D. Equbal, N. Dhiman, A. Singh, A. K. Kar, J. Shankar, S. Tehlan and S. Patnaik, Targeted Smart $\mathrm{pH}$ and Thermoresponsive N,O-Carboxymethyl Chitosan Conjugated Nanogels for Enhanced Therapeutic Efficacy of Doxorubicin in MCF-7 Breast Cancer Cells, Bioconjugate Chem., 2016, 27(11), 2605-2619.

66 S. Sornkamnerd, M. K. Okajima and T. Kaneko, Tough and Porous Hydrogels Prepared by Simple Lyophilization of LC Gels, ACS Omega, 2017, 2(8), 5304-5314.

67 J. Qu, X. Zhao, P. X. Ma and B. Guo, Injectable Antibacterial Conductive Hydrogels with Dual Response to an Electric Field and PH for Localized "Smart" Drug Release, Acta Materialia Inc, 2018, 72, 55-69.

68 R. H. Pelton and P. Chibante, Preparation of Aqueous Latices with $N$-Isopropylacrylamide, Colloids Surf., 1986, 20(3), 247-256.

69 Z. Wang, Y. Yu, W. Dai, J. Lu, J. Cui, H. Wu, L. Yuan, H. Zhang, X. Wang and J. Wang, et al., The Use of a Tumor 
Metastasis Targeting Peptide to Deliver DoxorubicinContaining Liposomes to Highly Metastatic Cancer, Biomaterials, 2012, 33(33), 8451-8460.

70 I. G. Economou and C. Tsonopoulos, Associating Models and Mixing Rules in Equations of State for Water/Hydrocarbon Mixtures, Chem. Eng. Sci., 1997, 52, 511-525.

71 G. H. Hudson and J. C. McCoubrey, Intermolecular Forces between Unlike Molecules. A More Complete Form of the Combining Rules. Trans. Faraday, Society, 1960, 56, 761-766.

72 A. S. L. Gouveia, C. E. S. Bernardes, L. C. Tomé, E. I. Lozinskaya, Y. S. Vygodskii, A. S. Shaplov, J. N. C. Lopes and I. M. Marrucho, Ionic Liquids with Anions Based on Fluorosulfonyl Derivatives: From Asymmetrical Substitutions to a Consistent Force Field Model, Phys. Chem. Chem. Phys., 2017, 19, 29617-29624.

73 M. Kotzabasaki, I. Galdadas, E. Tylianakis, E. Klontzas, Z. Cournia and G. E. Froudakis, Multiscale Simulations Reveal Irmof-74-Iii as a Potent Drug Carrier for Gemcitabine Delivery, J. Mater. Chem. B, 2017, 5, 3277-3282.

74 J. Goldwasser, J. S. Murray and P. Politzer, The Molecular Electrostatic Potential: A Tool for Understanding and Predicting Molecular Interaction, New Orleans Univ. LA Dept of Chemistry, 1998.

75 M. M. Talmaciu, E. Bodoki and R. Oprean, Global Chemical Reactivity Parameters for Several Chiral Beta-Blockers from the Density Functional Theory Viewpoint, Clujul Med., 2016, 89, 513.

76 J. Contreras-García, E. R. Johnson, S. Keinan, R. Chaudret, J.-P. Piquemal, D. N. Beratan and W. Yang, NCIPLOT: A Program for Plotting Noncovalent Interaction Regions, J. Chem. Theory Comput., 2011, 7, 625-632.

77 W. Shen, X. Chen, J. Luan, D. Wang, L. Yu and J. Ding, Sustained Codelivery of Cisplatin and Paclitaxel via an Injectable Prodrug Hydrogel for Ovarian Cancer Treatment, ACS Appl. Mater. Interfaces, 2017, 9(46), 40031-40046.

78 F. Haghiralsadat, G. Amoabediny, M. N. Helder, S. Naderinezhad, M. H. Sheikhha, T. Forouzanfar and B. Zandieh-doulabi, A Comprehensive Mathematical Model of Drug Release Kinetics from Nano-Liposomes, Derived from Optimization Studies of Cationic PEGylated Liposomal Doxorubicin Formulations for Drug-Gene Delivery. Artif. Cells, Nanomedicine, Biotechnol., 2018, 46(1), 169-177.

79 S. J. Owonubi, B. A. Aderibigbe, E. Mukwevho, E. R. Sadiku and S. S. Ray, Characterization and in Vitro Release Kinetics of Antimalarials from Whey Protein-Based Hydrogel Biocomposites, Int. J. Ind. Chem., 2018, 9(1), 39-52.

80 A. Upadhyay, R. Kandi and C. P. Rao, Injectable, SelfHealing, and Stress Sustainable Hydrogel of BSA as a Functional Biocompatible Material for Controlled Drug Delivery in Cancer Cells. ACS Sustain, Chem. Eng., 2018, 6(3), 3321-3330. 